\title{
The sleep-deprived human brain
}

Adam J. Krause ${ }^{1}$, Eti Ben Simon ${ }^{1}$, Bryce A. Mander ${ }^{1}$, Stephanie M. Greer ${ }^{2}$, Jared M. Saletin ${ }^{1}$, Andrea N. Goldstein-Piekarski ${ }^{2}$, and Matthew P. Walker ${ }^{1,2}$

${ }^{1}$ Department of Psychology, University of California, Berkeley

${ }^{2}$ Helen Wills Neuroscience Institute, University of California, Berkeley, California 94720-1650, USA

\section{Abstract}

How does a lack of sleep affect our brains? In contrast to the benefits of sleep, frameworks exploring the impact of sleep loss are relatively lacking. Importantly, the effects of sleep deprivation (SD) do not simply reflect the absence of sleep and the benefits attributed to it; rather, they reflect the consequences of several additional factors, including extended wakefulness. With a focus on neuroimaging studies, we review the consequences of SD on attention and working memory, positive and negative emotion, and hippocampal learning. We explore how this evidence informs our mechanistic understanding of the known changes in cognition and emotion associated with SD, and the insights it provides regarding clinical conditions associated with sleep disruption.

Without sleep, our cognitive and emotional abilities become markedly disrupted. What are the neural changes underlying these abnormalities? What do these alterations tell us about the pervasive link between sleep disruption and numerous neurological and psychiatric disorders?

There are at least three motivating reasons to build an accurate account of how sleep deprivation (SD) affects the human brain. First, from a neurobiological perspective, it is important to characterize which networks in the human brain are vulnerable or resilient to the effects of insufficient sleep and to understand how such SD-induced changes (such as regional or network increases or decreases in activity or changes in functional connectivity) explain the maladaptive changes in behaviour that are associated with SD. Of importance, SD does not simply represent the absence of sleep and the functions attributed to it. Rather, the sleep-deprived state is a composite of numerous detrimental factors, including extended wakefulness, as well as the absence of sleep. It is therefore insufficient only to develop an understanding of the functional benefits of sleep and then to reverse-infer an understanding of the neural and behavioural changes that would be expected following a lack thereof. Second, it is necessary to determine how comorbid sleep disruption - present in all major neurological and psychiatric conditions, including schizophrenia, Alzheimer disease, anxiety disorders and addiction disorders ${ }^{1,2}-$ contributes to or results from these disorders and

Correspondence to M.P.W. mpwalker@berkeley.edu.

Competing interests statement

The authors declare no competing interests. 
may thus be a target for disease treatment and/or prevention. Third, from a societal standpoint, such scientific evidence informs debates regarding sleep recommendations for both public and professional health policies in light of the acknowledged sleep-loss epidemic that now pervades industrialized nations ${ }^{3}$.

This Review seeks to move closer to these goals. We provide a focused overview of the impact of SD on the human brain across five functional domains: attention; working memory; positive, reward-related affect; negative affect; and hippocampus-dependent memory. Drawing on neuroimaging studies, we explore the neural signatures underlying phenotypic changes in cognition and affect following experimental SD. Moreover, we present examples of how these findings afford mechanistic insights into clinical disorders associated with disturbed sleep. The Review is necessarily focused on acute (24-48-hour) SD unless otherwise specified, as most neuroimaging studies to date are restricted to these time frames. Nevertheless, the issue of acute versus chronic sleep loss is discussed in more detail in BOX 1.

\section{Box 1}

\section{Acute versus chronic sleep deprivation and inter-individual variability}

A well-characterized feature of both acute sleep deprivation (SD) and chronic partial sleep restriction is a dose-dependent effect on task performance, especially in the domain of attention. The greater the amount and/or the longer duration of SD, the worse the accumulating attention deficit ${ }^{6}$. Thus, attentional impairment may be strongly coupled with extended wakefulness and with sleep pressure. These objectively measured impairments in performance are distinct from increases in subjective ratings of sleepiness following SD, which depend on the form of SD applied; for example, sleepiness increases as acute SD continues but does not always increase as chronic sleep restriction continues ${ }^{5}$. Thus, the physiological response to sleep loss may depend to some degree on the form of SD.

Regardless, the brain processes that mediate these cumulative deficits remain largely uncharacterized. For example, is there a brain region or set of brain regions involved in, or associated with, the tracking of sleep pressure ${ }^{131}$, or are there processes that track sleep pressure locally in a use-dependent manner within individual neuronal ensembles ${ }^{132}$ ? Sleep pressure-related molecules, such as adenosine, and the hypothalamic system governing the switch mechanism between sleep and wake ${ }^{133}$ are logical candidates for the chemical signalling and network mediation of this prototypical dose-dependent attentional impairment with sleep loss. In humans, neuroimaging analysis has revealed that association cortices, especially in the frontoparietal attention network, are particularly sensitive to sleep pressure, whereas subcortical thalamic and basal ganglia brain regions seem to be more affected by circadian processes ${ }^{19}$.

A second behavioural feature of SD that is poorly defined at the whole-brain level is a phenotypic difference in vulnerability to SD-associated cognitive impairments ${ }^{134-138}$. Approximately one third of participants show minimal impairments in sustained attention under SD conditions, and this seems to be a reliable, stable trait. By contrast, one third of participants manifest a severely vulnerable phenotype, showing marked attentional 
impairments. Moreover, it seems that differential vulnerability to the effects of acute sleep loss on attention is, in part, heritable ${ }^{137}$. Adding further complexity, the extent of an individual's cognitive impairment also depends on which cognitive domain is tested ${ }^{130}$. Thus, although performance impairments following SD within any given cognitive domain are highly reliable within the same individual, the degree of impairment is not necessarily reliable across cognitive domains within the same individual. The underlying brain correlates that differentiate these stable intra-individual and inter-individual differences are unclear, but the functional integrity of the frontoparietal attention network and genetic influences on sleep and circadian regulation are probably key factors ${ }^{139,140}$.

\section{Attention and working memory}

\section{Attention}

One cognitive ability that is especially susceptible to sleep loss is attention, which serves ongoing goal-directed behaviour ${ }^{4}$. Performance on attentional tasks deteriorates in a dosedependent manner with the amount of accrued time awake, owing to increasing sleep pressure $^{5-7}$. The prototypic impairments on such tasks are known as 'lapses' or 'microsleeps', which involve response failures that reflect errors of omission ${ }^{4-6}$. More specifically, attentional maintenance becomes highly variable and erratic (with attention being sustained, lost, reestablished, then lost again), resulting in unstable task performance ${ }^{4}$. Although the focus of this Review is sleep loss, it should be noted that daytime circadian alerting signals interact with SD, resulting in exponentially scaling attentional impairment with extended wakefulness (for reviews, see REFS 7,8). Nevertheless, the cumulative amount of extended time spent awake predicts lapses in attention with acute SD and with chronic partial sleep restriction (the latter occurring across many nights) ${ }^{5}$ (BOX 1). Why some individuals are more or less vulnerable to these attentional impairments following SD than others is less well understood.

An increasingly clear picture is emerging of how brain function related to attentional tasks is altered by acute SD. Reductions in functional MRI (fMRI) signal in the dorsolateral prefrontal cortex (DLPFC) and intraparietal sulcus while performing attentional tasks are a robust and reliable consequence of $\mathrm{SD}^{9-16}$ (FIG. 1). Indeed, sleep loss not only decreases task-related activity in these frontal and parietal regions but also diminishes activity in, and connectivity with, the extrastriate visual cortex during visuospatial attention tasks ${ }^{9-11}$. The behavioural consequences of these neural changes are reflected in deficiencies in attending to one specific stimulus while ignoring distractors ${ }^{10,11,17}$ or deficits in top-down allocation of attentional resources, such as orienting to a location where a target is expected to appear ${ }^{9,18}$. Beyond attentional focus at any particular moment, sleep loss impairs the capacity to sustain attention over time ${ }^{4}$. Here again, reductions in activity in the DLPFC and intraparietal sulcus contribute to attentional performance failure ${ }^{11-15,19}$.

In addition, thalamic activity during sustained attention is altered with $\mathrm{SD}$, suggesting that the thalamus could be an interacting node in this SD-affected network. However, the profile of activity change within the thalamus is not uniform. Some studies demonstrated greater activity under conditions of sleep loss ${ }^{11,16,20-23}$, whereas others have reported intermittent 
periods of diminished thalamic activity ${ }^{10,12}$. These discrepancies may be understood in the context of performance and of the cortical arousal that thalamic activity provides. When thalamic activity is elevated under conditions of sleep loss, attentional performance is frequently maintained but, when substantial reductions in thalamic activity are observed, lapses in attention are common $4,10,12,15$. The thalamus therefore represents a pivotal gating hub through which alterations in brainstem ascending arousal signals affect cortical attentional networks under SD conditions. Extant data therefore support a model in which the instability in ascending arousal-promoting input contributes both to the emergence of canonical attentional lapses during SD and the erratic, unpredictable expression of these lapses over time ${ }^{4,24}$ (FIG. 1). Notably, the observed reductions in thalamic activity are not present during attentional lapses in well-rested conditions ${ }^{12}$, suggesting that increased homeostatic sleep pressure may have a unique role in lapses following SD.

More recently, instability of the default mode network (DMN) has been implicated in attentional impairments with SD. Several reports describe an inability to fully disengage midline anterior and posterior cortical regions of the DMN during both selective and sustained attentional task performance under SD conditions ${ }^{13,16,25}$ (BOX 2; FIG. 1). Moreover, increased DMN activity during on-task performance for both sustained and selective attention tests was predictive of slower and less accurate performance by the participants ${ }^{16,25}$.

\section{Box 2}

\section{The unrested resting brain: sleep loss and resting-state activity}

In addition to inducing differences in task-related brain activity, sleep deprivation (SD) also affects resting-state brain connectivity. Resting-state networks are typically derived from the connectivity profile of spontaneous fluctuations in functional MRI (fMRI) signal and are thought to reflect the brain's intrinsic functional architecture that emerges without external task demands ${ }^{141,142}$. Across these networks, SD is associated with reduced connectivity within the default mode network (DMN), the dorsal attention network, and the auditory, visual and motor networks ${ }^{143-145}$. In fact, measures of abnormal change in whole-brain connectivity enable classification of an individual as either being rested or sleep deprived with more than $60 \%$ accuracy ${ }^{144}$.

Of the many different discrete resting-state networks, the DMN has demonstrated alterations under SD in two specific ways. First, connectivity between individual nodes of the DMN is decreased following one night of total $\mathrm{SD}^{143}$, especially between midline anterior and posterior nodes of the $\mathrm{DMN}^{146}$. Thus, SD degrades network integrity within the DMN, functionally uncoupling individual nodes. The second consequence of SD is the failure of the DMN to remain functionally distinct from other normally dissociable networks. Under normal rested conditions, the DMN is functionally decoupled from the brain's attentional networks - when the latter is engaged, the former is disengaged, and vice versa ${ }^{147}$. Both total SD and partial sleep restriction ${ }^{122,148-150}$ impair this adaptive decoupling, and this impairment is further associated with participants' cognitive vulnerability to $\mathrm{SD}^{143}$. 
The resting profiles of individual regions, specifically the amygdala and the thalamus, have also been examined following $\mathrm{SD}^{151,152}$. Similar to task-related connectivity changes (see FIG. 3), decreases in resting-state connectivity of the amygdala with regulatory and executive regions of the dorsolateral prefrontal cortex and anterior cingulate cortex have been demonstrated after 36 hours of SD. Conversely, resting-state connectivity of the amygdala with the posterior cingulate cortex and the precuneus increases after SD, possibly reflecting the role of these posterior midline cortical regions in regulating the balance between internally and externally directed cognition ${ }^{153}$. Resting-state connectivity between the thalamus and cortical regions is also generally decreased following sleep loss, especially thalamic connectivity with anterior and posterior cingulate regions of the $\mathrm{DMN}^{143}$ and with attentional and executive regions of the superior and medial prefrontal cortex ${ }^{151}$. As reductions in thalamocortical connectivity have also been demonstrated during sleep onset ${ }^{154}$, it remains possible that resting-state measures in sleep-deprived individuals capture mixed states of sleep and wake, comprising rapid transitions into sleep (known as microsleeps), while some degree of wake-like behavioural responding is nevertheless maintained ${ }^{155}$. This concern is emphasized by reports that have demonstrated reduced functional coupling between midline anterior and posterior nodes of the DMN under conditions of SD and once individuals have entered non-rapid eye movement sleep ${ }^{156}$. Overall, the limited evidence to date suggests that SD triggers reductions in the brain's intrinsic connectivity profile, ultimately leading to a breakdown of network integrity and a loss of adaptive functional segregation. These alterations may precede or exacerbate changes in task-related responses observed after sleep loss, and might predict the magnitude of cognitive and emotional impairments elicited by SD. A combination of task-related and resting-state fMRI studies of SD seems to be a fruitful avenue for future work, although accurate assessment of sleep or wake states inside the MRI scanner is warranted to assure the absence of microsleeps.

Why the sleep-deprived brain suffers this unstable 'flip-flop' gating between on-task functional activity and off-task DMN activity remains unclear. One candidate is abnormal activity of the right frontoinsular cortex, which controls the switch between DMN activity and task-related activity ${ }^{26}$. Consistent with this thesis, the salience-detection network, which includes the frontoinsular cortex, demonstrates reduced activity during the performance of attention tasks following sleep loss ${ }^{27}$. Together with changes in the frontoparietal attention network described above, these insula-based alterations may explain SD-induced impairments in attention to novel, salient targets ${ }^{28}$ and in the tracking of salient moving targets ${ }^{29}$. Nevertheless, trial-by-trial analyses of fMRI signal during salience-detection and attention tasks will be required to test this hypothesis.

\section{Working memory}

Working memory - the neural basis of which overlaps anatomically with the attention system - is also impaired by $\mathrm{SD}^{30,31}$. Deficits in both working- memory and attention tasks have been found to correlate with reductions in DLPFC and posterior parietal activity $^{20-22,32,33}$. As during attention tasks, fluctuations in thalamic activity ${ }^{20-22}$ and 
inappropriate perseverance of DMN activity are observed during working-memory task performance under SD conditions ${ }^{20,21,32}$. In addition, the degree of aberrant, on-task DMN activity predicts the severity of working-memory impairment in sleep-deprived individuals $^{20,21,32}$ (FIG. 1). Inappropriate gating of on-task relative to off-task network control may therefore provide one common mechanism underlying deficits in both attention and working memory caused by SD.

Also mirroring changes observed during attention tasks, alterations in thalamic activity and connectivity predict impairments in working-memory performance under SD conditions, most likely owing to the key role of the thalamus in cortical arousal. For example, increased connectivity between the hippocampus, the thalamus and the DMN predicts higher subjective sleepiness and is associated with worse working-memory performance under sleep-loss conditions ${ }^{34,35}$. By contrast, increased connectivity between the thalamus and the precuneus under SD conditions predicts greater recovery of working-memory performance, relative to the sleep-rested state. This finding is consistent with the hypothesis that, in the context of SD, the brain exhibits compensatory neural activity that can enable partial recovery of the performance of certain behaviours ${ }^{34,36}$.

Beyond reductions in activity in frontoparietal areas, extrastriate (visual) cortical regions also demonstrate reduced signal during visual working-memory task performance under conditions of sleep loss ${ }^{21,22,32}$. Recent evidence suggests a causal contribution of this regional decrease in task-related activation to performance deficits, as transcranial magnetic stimulation (TMS) targeted to the extrastriate cortex of sleep-deprived individuals improved visual working-memory performance, restoring it back to baseline levels ${ }^{37,38}$. Remarkably, repeated TMS application every 6 hours for 18 hours to extrastriate regions maintained working-memory performance for up to 3 days without sleep ${ }^{38}$. However, TMS stimulation intervention in the context of SD is not always consistent, and thus the reproducibility of such effects remains unclear ${ }^{39}$. Should a robust method for intervention emerge, it may have real-world relevance - for example, for professions in which attentive focus is crucial and $\mathrm{SD}$ is common (aviation or the military).

\section{Reward and incentive processing}

\section{Reward processing in the sleep-deprived brain}

The mesolimbic reward system is a network of interconnected brain regions, including the midbrain ventral tegmental area, striatum and regions of the PFC. The ventral tegmental area provides dopaminergic innervation to the striatum, which is connected to, and regulated by, areas of the PFC, particularly by the medial PFC (mPFC) and inferior orbitofrontal cortex (OFC) regions, guiding motivated actions and learning. This system has repeatedly been demonstrated to show sensitivity to SD, leading to alterations in motivated behaviours, such as risk taking, sensation seeking and impulsivity (FIG. 2).

In rats, SD disrupts dopaminergic function by modifying dopamine receptor sensitivity and availability in basal ganglia regions ${ }^{40,41}$. Humans deprived of sleep for one night show increases in ventral striatum activity in mixed monetary gamble task during the anticipation and receipt of monetary rewards ${ }^{42,43}$. Activity in affect-related regions in the frontal cortex 
that are associated with valuation and viscerosensory functions, including the insula and $\mathrm{mPFC}$, is also substantially increased following $\mathrm{SD}^{42,43}$.

Together, these findings suggest that subcortical reward-related regions of the brain, together with related cortical regions coding salience and valuation, seem to become hypersensitized by the state of acute SD. However, the degree to which fMRI signal amplitude tracks reward magnitude in these subcortical and cortical regions does not seem to differ between rested and deprived conditions ${ }^{44}$. Rather, SD triggers a generalized increase in reward sensitivity that impairs reward discrimination accuracy, such that the brain becomes less capable of accurately coding incremental increases in reward value, from low to high.

Consistent with this thesis, fMRI signal in the mPFC, OFC and anterior insula cortex in sleep-deprived individuals does not accurately discriminate between trials involving monetary reward and punishment values and trials involving no monetary reward or punishment ${ }^{42,43}$. This inaccurate representation of reward value within frontal regions is similarly observed during the outcome phase of incentive decision trials ${ }^{42,43}$, suggesting a further failure to update accruing reward history and probability. Inaccurate coding of reward and/or punishment valence in the PFC following sleep loss may therefore prevent the ability to update changing incentive value (weights) of reinforcing stimuli over time ${ }^{42,43}$ (FIG. 2). This would further contribute to non-optimal reward-dependent decision making and actions. Fitting this profile, sleep-deprived individuals performing the Iowa Gambling Task make more-risky decisions and assign greater weights to more-recent rewards ${ }^{45,46}$. This pattern indicates a failure to appropriately integrate rewards and their accumulating value over time following SD, reflecting temporally shorter -sighted updating of rewards.

In addition to deficits in the activation of frontal reward circuits following SD, studies have revealed that responses of the striatum and amygdala to emotionally pleasurable or hedonic images ${ }^{47}$, as well as desirable food stimuli ${ }^{48}$, are amplified with SD. Moreover, the same studies reported failures of discriminatory signalling of stimulus valence (for example, discriminating desirable from undesirable foods) in the anterior insula, $\mathrm{mPFC}$ and $\mathrm{OFC}$, of sleep-deprived participants. Furthermore, sleep- deprived individuals were more likely to rate neutral pictures as positive ${ }^{47}$ or to express a greater desire for high-calorie foods ${ }^{48}$. That is, participants fail to accurately disambiguate non-rewarding from rewarding stimuli when sleep deprived, shifting towards an over-generalized reward bias, even for normally lessrewarding stimuli.

Although current results of reward processing point to effects in the mPFC, OFC and insular cortex, together with the basal ganglia, other affective regions, such as the limbic regions of the medial temporal lobe and executive lateral regions of the PFC, should not be discounted. Equally important, not all human SD studies have reported amplified basal ganglia activity during reward-related decision making ${ }^{49}$, an inconsistency we return to below.

Beyond monetary reward processing, another behavioural feature sensitive to a lack of sleep is impulsivity. Impulsivity is a multifactorial construct, and different types of impulsivity can manifest in behaviourally different ways. Nevertheless, impulse control is central to many 
incentive-based decision processes ${ }^{50,51}$. Changes in impulsivity may therefore contribute to alterations in reward decision making and risk taking that occur following SD.

In tasks that require either cued motor response execution or withholding, often referred to as a Go/No-Go task, participants with either partial sleep restriction (6 hours of sleep per night for 4 consecutive nights) or one night of total sleep deprivation showed considerably lower response inhibition and cognitive control, produced more frequent errors of inhibition, and exhibited slower learning of cue-incentive associations ${ }^{52-56}$ (FIG. 2).

However, in delay-discounting tasks, which require participants to choose between small, immediate rewards relative to large, delayed rewards, SD does not seem to alter impulsivity - a lack of an effect that is similarly observed in probabilistic discounting tasks, in which participants choose between small but certain rewards and large but uncertain rewards $49,53,54,57$. Similarly, total acute SD and partial sleep restriction (4 nights of 6 hours of sleep per night) do not affect delay discounting or probabilistic discounting ${ }^{54}$. However, measures of effort discounting (whereby rewards are discounted according to the effort required to attain them) are affected by SD. Under conditions of sleep loss, low-effort rewards elicit even greater reward-related brain activity than high-effort rewards, relative to sleep-rested conditions ${ }^{49}$.

Similarly, inconsistent effects of SD have been reported using the Balloon Analogue Risk Task (BART) - a standard test of reward-seeking, risk-taking behaviour that involves impulse control. Partial sleep restriction (1 night of 5 hours of sleep) results in a significant increase in the tendency to overinflate a computerized balloon to obtain more reward ${ }^{54,58}$. By contrast, total SD for a single night resulted in no change in BART performance by male participants but a decrease in risky overinflation by female participants ${ }^{53}$ (for a review, see REF. 59). Studies that focus on this and other specific features of impulsivity, such as response withholding, while systematically keeping other factors constant (for example, reward magnitude, reward delay and sex) are necessary to develop a clearer understanding of sleep loss and risk-taking impulsivity.

Inconsistencies in the effects of SD on incentive processing are not limited to the domain of impulsivity. Some reports using incentive tasks that do not involve impulsivity (wherein participants simply expect or receive rewards and/or punishments without needing to resist impulsive responses) have also failed to show significant differences in mesolimbic brain activity and/ or behaviour caused by $\mathrm{SD}^{44,49,57}$. Instead, these studies only demonstrate effects on an individual-participant level ${ }^{44,49,57}$. One possible explanation is that some studies employ mixed gambles, combining gains and losses in the same trial. This may limit the ability to identify differences in processing of gains or losses independently ${ }^{60}$. A second possibility is that individual differences may interact with the effects of $\mathrm{SD}$, thus obscuring group-level significance if not explicitly considered. A recent study ${ }^{60}$ found that individuals expressing a variant of the dopamine transporter that was previously associated with differences in synaptic dopamine availability showed different striatal reward responses following sleep loss. Unlike the control group, only participants with trait-elevated synaptic dopamine exhibited greater striatal responses during reward anticipation following SD than when well rested. This suggests that phasic dopamine availability is increased in these 
individuals, thereby elevating SD-associated increases in the reward responsivity of the nucleus accumbens. Thus, effects of sleep loss on reward processing seem to be sensitive to several interacting factors, including sex and trait genetics.

\section{Sleep deprivation and dopamine function}

With these nuances taken into account, studies to date nevertheless indicate that SD significantly increases the tendency of reward sensitivity, risk taking and impulsivity, and disrupts reward-value updating and integration. One possible mechanism that might explain these increases in approach and consummatory behaviour is altered dopamine signalling, which is associated with extended wakefulness perhaps more than with the absence of sleep itself. Several lines of evidence support this dopaminergic framework (FIG. 2).

First, dopamine is associated with arousal; innately higher levels of dopamine predict lower sleep propensity ${ }^{61}$. Second, rodent studies have established that wake-promoting stimulant drugs such as amphetamine seem to operate in part by blocking dopamine metabolism, thereby increasing dopamine transmission and thus arousal. Third, depleting catecholamines, including dopamine, reduces wake propensity, lowering vigilance and inducing sleep ${ }^{62}$ (although interestingly the antihypertensive and antipsychotic reserpine, which reduces catecholamine function, has nominal effects on sleepiness ${ }^{63}$ ).

Changes in dopamine receptors may also contribute to alterations in reward-driven behaviour following SD. Positron emission tomography (PET) ligand studies have reported that one night of total SD downregulates the availability of dopamine D2 and D3 receptors (D2/3Rs) in both the dorsal striatum (which includes the caudate and putamen) and ventral striatum ${ }^{40}$ (FIG. 2), consistent with earlier PET studies showing general hypometabolism in the basal ganglia following $\mathrm{SD}^{64}$. PET-assessed downregulation of D2/3Rs in the ventral striatum predicted fMRI-measured decreases in thalamic activity during an attentional $\operatorname{task}^{65}$, as did the degree of generalized hypometabolism in the basal ganglia after $\mathrm{SD}^{64,66}$. Besides the relevance of this change for objective attention and working -memory performance, the degree of $\mathrm{D} 2 / 3 \mathrm{R}$ downregulation similarly predicts subjective sleepiness under SD conditions ${ }^{40}$. This result suggests that sleep pressure might be indexed by the levels of different dopamine receptors; consistent with this, adenosine accumulates with increasing time awake, activating $\mathrm{A}_{2 \mathrm{~A}}$ receptors and thereby driving $\mathrm{D} 2 \mathrm{R}$ internalization ${ }^{40,67}$. Moreover, agonists of the $\mathrm{A}_{2 \mathrm{~A}}$ receptor and $\mathrm{D} 2 \mathrm{R}$ demonstrate allosteric interaction such that $\mathrm{A}_{2 \mathrm{~A}}$ receptor agonists decrease the affinity of D2Rs for their agonists, including dopamine ${ }^{68}$. Thus, adenosine accumulation, which is associated with increased time spent awake, may decrease D2/3R activity by at least two possible mechanisms: by increasing D2/3R internalization and by reducing binding of dopamine to D2Rs (FIG. 2).

That SD is associated with a downregulation of D2/3Rs may initially seem to contradict the SD-associated increases in neural and behavioural reward sensitivity described above. However, one tenable (and experimentally testable) hypothesis that may reconcile this paradox involves D1Rs. Specifically, decreases in D2/3Rs could result in an imbalance of dopamine receptor availability, leading to the remaining D1Rs becoming disproportionately stimulated by the same amount of presynaptically released dopamine (FIG. 2). Outside the context of SD, in rodents, this imbalance causes greater approach behaviour to food 
rewards ${ }^{69}$ and increases the addictive potential of cocaine ${ }^{70}$ (behavioural consequences that, notably, have both been independently associated with $\mathrm{SD}^{71,72}$ ). Furthermore, the increase in striatal fMRI signal elicited by reward incentives under SD conditions seems to principally reflect D1R action ${ }^{73}$. Thus, SD-associated decreases in D2/3R availability may, by indirectly increasing dopamine binding to the remaining D1Rs, increase approach-driven and rewarddriven behaviour and fMRI-indexed striatal activity (FIG. 2). The neural and behavioural consequences of sleep loss on incentive processes also offer mechanistic and therapeutic insights into the conditions of obesity, addiction and substance use (BOX 3).

\section{Box 3}

\section{Clinical insights concerning sleep loss and emotion}

\section{Positive emotion}

The interaction between sleep deprivation (SD) and dopaminergic reward functioning is relevant for clinical conditions involving sleep disruption and dysfunction of reward or dopamine signalling. In alcohol addiction, $\sim 60 \%$ of alcohol-addicted individuals seeking treatment complain of insomnia symptoms in the 6 months leading up to intervention, and more than half of these individuals report using alcohol under the (erroneous) assumption that it helps to regulate sleep ${ }^{157}$. In addition, alcohol-addicted individuals who report insomnia are more likely to relapse than those without sleep problems ${ }^{157}$. Thus, sleep loss seems to be an important factor in the development and/or maintenance of addiction disorders associated with changes in mesolimbic dopamine function and a vulnerability factor predisposing individuals to fail in efforts of abstinence. Of concern, sleep disruption in childhood predicts subsequent substance use in later adolescence stages $^{158}$.

There is now also considerable epidemiological evidence linking sleep disruption and obesity ${ }^{159}$. Experimental work has characterized the associated causal mechanisms, including changes in the appetite-regulating hormones leptin and ghrelin, and in the central brain mechanisms that regulate appetite and food choice selection ${ }^{160}$. Decreased activity in decision-regulating regions of the frontal cortex following SD, combined with enhanced subcortical mesolimbic sensitivity, leads to increased desire for, and selection of, high-calorie food items ${ }^{48}$. Indeed, compared with a sleep-rested state, experimental sleep restriction increases calorie consumption and promotes weight gain ${ }^{161}$.

Notably, the alterations in brain function that have been associated with obesity overlap with those observed in drug addiction ${ }^{162}$; for example, a reduction of dopamine D2 and D3 receptors is observed in both. Such findings warrant a deeper consideration of insufficient sleep as a possible predisposing factor underlying obesity and drug addiction. If true, targeted sleep restoration may aid by re-engaging neural mechanisms that govern adaptive food choices and craving, respectively.

\section{Negative emotion}

A recent model has proposed that sleep loss prevents the normal overnight lowering and thus restoration of central adrenergic signalling that normally occurs during rapid eye movement (REM) sleep, leading to heightened noradrenergic tone and thereby an over- 
generalized responsivity within select affective salience networks ${ }^{84}$. The translational ramifications of this model are well exemplified in post-traumatic stress disorder (PTSD), which is associated with sleep disruption and fragmentation, decreases in total and REM sleep duration ${ }^{163-165}$, and nocturnal hyperarousal ${ }^{166-168}$. In parallel, people with PTSD show marked alterations in noradrenergic activity ${ }^{163}$, including the lack of normal overnight reduction in noradrenaline levels ${ }^{169}$. The REM-sleep model may help to understand these co-occurring features. Specifically, decreases in night-time, total and REM sleep quantity, and increases in high-frequency electroencephalogram (EEG)assessed activity (associated with hyperarousal) during REM sleep, are proposed to contribute to daytime hyperadrenergic tone in PTSD. Moreover, persistent hyperadrenergic activity during wakefulness could reduce subsequent night-time sleep quantity and quality, producing a vicious cycle. Crucially, this cycle could lead to overgeneralized emotional sensitivity within the affective salience network of the brain that is innervated by noradrenaline, including the amygdala, and the viscerosensory regions of the insula and anterior cingulate cortex, as already reported in a meta-analysis of functional MRI studies in PTSD ${ }^{89}$. This same model could explain how nocturnal use of prazosin - an a 1-adrenergic receptor antagonist commonly prescribed for PTSD - can, in some cohorts, restore the duration and quality of total and REM sleep, and decrease PTSD symptoms ${ }^{170-173}$. Our framework may also account for the excessive emotion reactivity and impaired emotional discrimination observed in PTSD $^{174-176}$, and the maladaptive generalization of fear responses to commonly non-threatening stimuli ${ }^{174-176}$. Interestingly, PTSD-like symptomatology was experimentally induced by selective deprivation of REM sleep in healthy individuals; this manipulation resulted in increased autonomic sensitivity to previously extinguished conditioned stimuli (that is, heightened sensitivity) and impaired autonomic discrimination of conditioned from safety signals (decreased emotional specificity) ${ }^{177-179}$. Conversely, the amount and EEG quality of REM sleep predicts participants' ability to accurately discriminate between threat and safety signals (indicating improved emotional specificity) ${ }^{180}$.

\section{Aversive stimulus processing}

Sleep loss reliably triggers changes in negative (aversive) emotional processing, including irritability, emotional volatility, anxiety and aggression ${ }^{56,74-77}$, as well as suicidal ideation, suicide attempts and suicide completion ${ }^{78-80}$. These findings suggest that SD alters specific process domains, including basic affective reactivity, as well as emotional discrimination and emotional expression, which are more complex.

\section{Aversive stimulus response}

Early neuroimaging studies focused on the effects of SD on responsivity to rudimentary aversive stimuli; for example, one night of SD was shown to result in a $60 \%$ increase in amygdala reactivity to negative images, such as weapons, venomous snakes and spiders, and mutilations ${ }^{81}$ (FIG. 3). This signature of amygdala hyper-reactivity has since been replicated following sleep restriction (4 hours of sleep per night for 5 nights) ${ }^{82}$ and in individuals with poor sleep quality, as assessed at their homes ${ }^{83}$. Notably, amygdala hyper-reactivity has been 
reported during rapid, subconscious viewing of faces expressing fear after sleep restriction ${ }^{82}$, indicating that this amplified limbic reactivity can occur subliminally, independent of conscious, deliberative cognition ${ }^{82}$.

One possible mechanism underlying such amygdala hypersensitivity involves a loss of regulatory control, resulting in contextually inappropriate amygdala reactivity ${ }^{84}$. Decreases in functional connectivity between top-down control regions of the mPFC and the amygdala have been reported following a night of total SD or 5 nights of partial sleep restriction (4 hours sleep per night), as well as in participants reporting less than 6 hours of habitual sleep ${ }^{81-83,85,86}$ (FIG. 3).

SD further alters the brain anticipation of cued emotional experiences. For example, one night of SD elevates cue-evoked activity in the amygdala, anterior insula and anterior cingulate cortex in anticipation of impending emotional picture slides, similar to those described above ${ }^{87}$, and also increases anticipatory responses of the peripheral autonomic nervous system ${ }^{88}$.

Interestingly, inter-individual differences in trait anxiety levels predict the size of these SDinduced increases in anticipatory brain activity, with highly trait-anxious individuals expressing the largest increases in pre-emptive anterior insula activity ${ }^{87}$. The latter result is of clinical relevance to anxiety disorders (which have been associated with apprehensive worry about future events) for at least three reasons: first, highly trait-anxious individuals are already those at greatest risk for developing an anxiety disorder; second, excessive anticipatory insula activity is a characteristic of most anxiety disorders ${ }^{89-91}$; and third, sleep disruption is a recognized symptom of anxiety disorders ${ }^{92}$.

\section{Emotion discrimination and expression}

An additional phenotype of the sleep-deprived human brain emerges when participants are challenged with the more complex task of disambiguating between varied emotional signals. In sleep-rested participants, regions of the amygdala, insula and cingulate that are associated with salience detection can discriminate between stimuli of different emotional strengths. By contrast, in sleep-deprived participants, these regions demonstrate a saturated and flattened response to emotional stimuli. Sleep-deprived individuals therefore express a generalized excess of emotional sensitivity, with impairment in emotional discriminatory specificity ${ }^{84}$ (FIG. 3). For example, sleep-deprived individuals are less accurate at rating facial expressions within the moderate range of emotional strength ${ }^{93}$ and rate neutral images as more emotionally negative ${ }^{94}$. SD further impairs the accurate discrimination of threatening (antisocial) from affiliative (pro-social) facial signals ${ }^{95}$, again promoting an overall bias towards increased (inaccurate) perception of negative threat.

Recent work has begun to uncover the neural basis of these impairments in emotional discrimination (FIG. 3). Consistent with the failure to accurately code positive, reward incentive signals (described above), one night of SD impairs the discrimination of faces expressing negative emotions by viscerosensory regions of the anterior insula and anterior cingulate cortices, and to a degree, the subcortical amygdala ${ }^{95}$. Similarly, sleep loss results 
in generalized, nonspecific increases in amygdala activity (as measured by fMRI or electroencephalography) in response to aversive and neutral emotional pictures ${ }^{96,97}$.

These findings have led to the proposal that SD causes a state of central and peripheral emotional hypersensitivity that prevents emotional reactivity from being appropriately graded $^{84}$. As a result, SD impairs the afferent-efferent communication between the brain and body, which is crucial for the 'embodied' mapping of, and disambiguation between, different emotions. The consequence is a behavioural phenotype of indiscriminate emotional generalization, or blunting, consistent with poor signal-to-noise balance during emotional processing $^{84}$ (FIG. 3).

Such blunting may disrupt not only the internal mapping of an individual's own affective state but also the ability to simulate the feelings of others. Supporting this hypothesis, sleepdeprived individuals and habitually poor sleepers demonstrate impaired empathetic sensitivity, which involved sensing the emotion state of individuals in picture slides ${ }^{98,99}$. Sensing the internal homeostatic status of the body, and thereby tracking one's own affective state, requires interoceptive processing within a network that includes the insula, mPFC and amygdala ${ }^{100}$. Sleep loss degrades the sensitivity of this network, leading to a shift of the homeostatic set point used to register, and differentiate between different levels of, emotional salience ${ }^{95}$. In an emotional face discrimination task, sleep-deprived individuals showed overgeneralized activity in viscerosensory brain regions and impaired autonomic cardiac discrimination (indexed using moment-by-moment changes in heart rate) ${ }^{95}$. Compared with when they were rested, individuals who were sleep deprived also showed a weaker correlation between the activity of the central and peripheral autonomic (cardiac) systems. Thus, SD compromises the faithful discrimination of emotional signals within higher-order emotional brain regions by inducing network hypersensitivity and, in tandem, disrupts the 'embodied' reciprocity between viscerosensory central and peripheral autonomic processing of complex social signals. As a result, there can be a loss of sensitivity in detecting, and accuracy in recognizing, emotions.

It is possible, although currently untested, that this same disruption within and between central and peripheral autonomic nervous system networks explains why sleep-deprived adults ${ }^{101}$ and infants deprived of a day-time nap ${ }^{102}$ show incongruent or even absent outward expression of emotions (FIG. 3). Individuals restricted to 4 hours of sleep for a single night also demonstrate a slowing of reactive facial expressions in response to emotional stimuli ${ }^{103}$. Emotional vocalizations following short-term sleep restriction have similarly been reported to be blunted ${ }^{104}$.

Thus, multiple modes of emotional expression are diminished by insufficient sleep; a feature pertinent considering that emotional expressions are all influenced in some manner by the autonomic nervous system, specifically the vagal system ${ }^{105}$. This same explanatory model of central-peripheral autonomic decoupling may offer further mechanistic insights into the recognized associations between reduced sleep and decreased interpersonal functioning ${ }^{108}$, increased antisocial interactions ${ }^{109}$, a lower understanding of relationship concerns (leading to greater interpersonal conflict) ${ }^{110}$ and, in children and adolescents, increased peer-related 
problems, such as hyperactivity and inattention, conduct problems, and peer disagreement ${ }^{106,107}$.

Less understood are the cellular and molecular mechanisms underlying the emotional brain and body dysfunction that is caused by a lack of sleep. One proposed mechanism has centred on the heightened noradrenergic tone from the locus coeruleus ${ }^{84,111,112}$; according to this hypothesis, sleep loss prevents the normal overnight reduction in noradrenergic tone that usually occurs during rapid eye movement (REM) sleep ${ }^{112-114}$. The resulting hypernoradrenergic tone may thus lead to heightened and over-generalized responsivity within the affective salience network that is innervated by noradrenaline: the amygdala and the viscerosensory cortical regions ${ }^{84}$. Consequently, there may be a loss of specificity in affective signalling within this network, possibly helping to explain the SD-associated inaccuracy in emotional discrimination. This REM sleep model ${ }^{84}$ offers several testable predictions regarding the reliable co-occurrence of sleep disruption (including REM sleep disruption) in clinical anxiety disorders (BOX 3). However, noradrenaline alone is unlikely to be the sole neurochemical factor underlying the affective dysregulation caused by SD. Integrating changes in other sleep-dependent neurochemicals, such as dopamine, may provide a more accurate explanatory model.

\section{Hippocampal memory processing}

Many studies have characterized the beneficial impact of sleep on the post-learning, offline consolidation of hippocampus-dependent memories (for a review, see REF. 115). By contrast, few studies have explored the adverse effects of SD on this process ${ }^{115}$; instead, the majority of such neuroimaging investigations have examined the impact of sleep loss on initial hippocampus-dependent memory encoding, on which we focus here.

In rodents, SD substantially decreases the ability to induce hippocampal long-term potentiation (LTP; an electrophysiological measure of neuroplasticity) and, if LTP is induced, the enhancement subsequently decays more rapidly ${ }^{116}$. Further, sleep loss reduces hippocampal synthesis of proteins associated with neuroplasticity and impairs hippocampal neurogenesis ${ }^{117}$.

The extracellular build-up of the metabolic product adenosine during extended wake affects plasticity. High levels of adenosine disrupt intracellular cAMP signalling in rodents and decreases hippocampal AMPA and NMDA receptor signalling; all of which are necessary for stable LTP ${ }^{115}$. Given that adenosine is cleared from the brain during sleep, these effects on plasticity may result from two mutually non-exclusive reasons: abnormally extended wake or a loss of sleep.

Building on these findings, early human neuroimaging and behavioural studies established that one night of SD impairs learning and encoding-related activity within the medial temporal lobe ${ }^{118}$, specifically the hippocampus ${ }^{36}$ (FIG. 4). Moreover, selective deprivation of non-rapid eye movement (NREM) slow-wave sleep using auditory stimulation, which preserves total sleep time, also lowers encoding-related activity in the hippocampus and associated learning ${ }^{119}$. Conversely, enhancing the power of NREM slow-wave activity, using 
transcranial stimulation, increases hippocampal learning ability after sleep ${ }^{120}$, further supporting a causal role for slow-wave sleep in hippocampal memory encoding.

In addition, SD alters learning-related hippocampal connectivity. Functional coupling between the hippocampus and perceptual regions of the occipital (visual) cortex and nearby regions in the medial temporal lobe during visual episodic-memory encoding is decreased under SD conditions ${ }^{36}$ (FIG. 4). By contrast, SD is also associated with increases in hippocampal connectivity with subcortical arousal regions, including the brainstem and the thalamus $^{36}$ (FIG. 4). The latter observation has been interpreted as reflecting a compensatory effort to mobilize basic arousal networks, albeit insufficient to rescue behavioural performance ${ }^{36}$.

A recent report has demonstrated that the structural morphology of the human hippocampus, and specifically the volume of the CA3-dentate gyrus subfield region, predicts the degree of vulnerability to the impact of SD on memory encoding ${ }^{121}$. Moreover, this same measure also predicted the amplitude of NREM slow-wave oscillations during recovery sleep and, by way of this mediating influence, determined how well memory-encoding ability was restored when assessed again after recovery sleep. Thus, human hippocampal subfield anatomy may represent a novel trait-like factor and potential biomarker of susceptibility of memory processing to, and recovery from, the disrupting effects of SD.

The hippocampus does not operate in isolation during memory encoding, however, but acts in a broad network of anatomically and functionally connected cortical regions. Some of these are proposed to participate in encoding memory representations themselves (for example, sensory perceptual regions), whereas others are involved in operations that support the act of encoding, such as the DLPFC and posterior parietal regions, which are required for directed attention ${ }^{32}$. Consistent with this, human neuroimaging studies have revealed that $\mathrm{SD}$ is associated with larger-network impairments that co-occur or are functionally linked with deficits in hippocampus-dependent encoding of new memories. For example, SD for 24 hours decreases activity in regions of the DLPFC and posterior parietal cortex required for goal-directed attention during a visual episodic- memory encoding task ${ }^{122}$. Reductions in the activity of the scene-selective fusiform cortex following SD are less clear to interpret and may reflect impairment in the functional role of the visual cortex in processing visual scenes and/or the failure of the top-down control of attention by frontoparietal networks ${ }^{123}$. Nevertheless, the size of the alterations in activity of all three of these cortical regions (frontal, parietal and occipital) predict the degree of memory impairment under SD conditions and the severity of attentional task lapses.

As with working-memory and selective attention tasks, significant differences in DMN activity within cortical regions have been reported during episodic-memory encoding following $\mathrm{SD}^{124}$. Indeed, the size of reductions and increases in the activity of the anterior cingulate and precuneus (which are both nodes of the DMN), respectively, discriminated sleep-deprived from sleep-rested participants with $93 \%$ sensitivity and $92 \%$ specificity ${ }^{124}$. Unstable control of the DMN might therefore be a common neural phenotype underlying deficits in various cognitive tasks (including attention, working memory and episodic memory) in the sleep-deprived human brain (BOX 2). 
Only one study to date has examined the under lying neurochemical basis of memoryencoding deficits associated with SD. Administration of an acetylcholinesterase inhibitor, which prolongs the central synaptic effects of acetylcholine, partially restored activity in frontoparietal and fusiform regions in sleep-deprived participants towards levels observed under sleep-rested conditions ${ }^{122}$ (FIG. 4). Interestingly, the greatest benefit of this acetylcholinesterase inhibitor treatment was observed in those individuals who, with SD, demonstrated the largest decreases in brain activity during a semantic judgement task and the worst performance in word recognition. That is, those most susceptible to sleep loss on these tasks were those who recovered most function after being treated with the drug.

Given that acetylcholinesterase inhibitors enhance attentional processes that support episodic memory ${ }^{125}$ and directly affect sensory processing ${ }^{126}$, some part of this benefit might be mediated by cholinergic influences on arousal. An alternative or additional plausible hypothesis is that the actions of the inhibitor facilitate hippocampal plasticity mechanisms that consequentially increase hippocampal engagement of sensory and attentional networks in the cortex. Accordingly, acetylcholinesterase inhibitors enhance activation of the visual and parietal cortices in sleep-deprived individuals during a visual short-term-memory task and, compared with placebo, improve task performance ${ }^{127}$.

At a translational level, impaired hippocampus-dependent memory encoding following sleep loss is relevant to several disorders and conditions that involve deficiencies in both sleep and memory encoding. Two such examples are ageing and dementia. Older, cognitively normal adults show marked impairments in NREM sleep, and these deficits are magnified in size in Alzheimer disease ${ }^{128}$. Disruptions of NREM sleep and associated reductions in slow-wave activity and sleep spindles in older adults and, even more dramatically, in patients with Alzheimer disease predict reductions in encoding-related activity in the hippocampus on the following day, which, in turn, predict the degree of learning impairment ${ }^{119,129}$. Sleep disruption therefore may contribute to cognitive decline, and especially impairments in hippocampus-dependent memory processing, with ageing. This evidence raises the possibility that sleep restoration may be a novel therapeutic intervention, as well as a midlife preventative measure against age-related decline in hippocampus-dependent memory (for a more detailed discussion, see REF. 128).

\section{Conclusions}

Several insights emerge from this Review. First, SD triggers a complex set of bidirectional changes in brain activity and connectivity - depending on the specific functional operation and anatomical regions in question. Equally important, however, are moment-to-moment fluctuations in brain activity that occur during performance across the timescale of minutes, reflecting a neural phenotype of regional and network instability. This is especially apparent in the domains of attention and working memory. Second, the heterogeneous array of brain changes (averaged and moment-to-moment) results in an equally diverse set of disruptions in human behaviour across nearly all domains of cognition and affect. Nested within this statement is another related subtlety: not all changes in brain function that are associated with sleep loss are maladaptive and thus represent deficiencies. Instead, some aspects of these neural alterations seem to correlate with preserved task performance, indicating that 
they may be compensatory and thus adaptive. That the human brain can compensate for SD to a certain degree seems tenable, given that individuals use compensatory effort and behavioural strategies (for example, talking or standing) when attempting to stay awake when sleep deprived. However, the extent and duration of compensatory brain function, which networks and associated operations they are in, and which behaviours are maintained as a consequence, remain poorly characterized. For example, do the effects of SD depend on interactions among hierarchies of functional brain networks, such that deficits or compensation in a fundamental, base-level network (for example, the attention network) dictates respective impairment (or compensation) in higher-level, dependent operations (such as intentional memory encoding or conscious emotional processing)?

Equally important, this Review reveals our current inadequacies of knowledge regarding the sleep- deprived human brain. For example, we have remarkably little understanding of the whole-brain consequences of long-term, chronic sleep loss, as most studies have focused on acute, total SD (BOX 1). Such knowledge would be crucial considering that chronic partial sleep restriction, from weeks to years, is representative of (and thus relevant to) the sleep deficiency observed in the developed world. We also lack any comprehensive understanding of whether and how human brain networks may recover from acute and chronic sleep restriction. Are the underlying neural mechanisms that support recovery from these different forms of sleep loss the same, partially overlapping, or entirely separable? Relatedly, does neural recovery occur across the same time period or over a different temporal duration following different forms and doses of sleep loss? What types of recovery sleep stages and/or aspects of sleep physiology transact functional brain restoration following sleep loss? These questions are important from a societal and public-health perspective, both in professional circumstances in which the privation of sleep is rife (for example, in the military, in aviation and in medicine) and in subtler forms (for example, the public trend of short sleep durations during the week followed by longer sleep durations at weekends).

Another key unresolved issue extends beyond the phenotypic between-participant differences in vulnerability to the neural and behavioural effects of SD within a cognitive domain (such as attention) (BOX 1) and centres on emerging evidence suggesting withinparticipant, between-domain differences in vulnerability: an individual who is resilient to the effects of SD in one functional domain (such as attention) may conversely be vulnerable to that same dose of sleep loss in other functional domains (such as memory or emotion processing $)^{130}$. This suggests that different brain networks in the same individual may be differentially susceptible to sleep loss. These hypothetical within-subject differences in neural network vulnerability and resilience to SD have not yet been experimentally tested.

Finally, the basic scientific findings regarding sleep loss have not yet been routinely applied in the clinic, despite the examples that we describe in BOX 3. Sleep abnormalities are robustly observed in every major disorder of the brain, both neurological and psychiatric. Sleep disruption merits recognition as a key relevant factor in these disorders at all levels, from diagnosis and underlying aetiology, to therapy and prevention. More collaborative work between basic and clinical scientists in the field will be necessary to accomplish this goal. Notably, the answers to all these questions have perhaps never been more pressing 
considering the professional, societal and clinical implications that continue to scale in lockstep with the precipitous decline in sleep duration throughout industrialized nations.

\section{Acknowledgments}

This work was supported by awards R01-AG031164, R01-AG054019, RF1-AG054019 and R01-MH093537 to M.P.W. from the US National Institutes of Health.

\section{Glossary}

\section{Sleep disruption}

Abnormal sleep that can be described in measures of deficient sleep quantity, structure (reflected by, for example, sleep cycle architecture) and/or sleep quality (assessed using, for example, spectral electroencephalogram power).

\section{Functional connectivity}

In functional MRI, the statistical association between time series of blood-oxygen-leveldependent signal in two or more anatomically distinct brain regions.

\section{Attention}

The ability to selectively maintain focus on behaviourally relevant stimuli, while disregarding irrelevant stimuli.

\section{Working memory}

The ability to maintain, manipulate and integrate mental representations of relevant information even when it is no longer present in the environment.

\section{Sleep pressure}

Also called 'homeostatic sleep drive' or 'Process S'. A set of homeostatic neurobiological processes that increase the likelihood of sleep, or 'sleep propensity'.

\section{Partial sleep restriction}

The state associated with a reduction (but not total absence) of sleep in the prior night or nights, usually ranging from 1-6 hours of sleep reduction. Sleep restriction is chronic if it persists for more than 24 hours.

\section{Default mode network}

(DMN). Collection of brain areas, including midline frontoparietal regions, that usually disengage during externally driven, goal-directed task performance and re-engage upon task termination.

\section{Frontoparietal attention network}

A bilateral brain network with core regions in the frontal and parietal lobes that exerts topdown control of sensory cortex to bias stimulus processing.

\section{Impulsivity}

Acting without deliberation, or choosing short-term gains over long-term gains.

\section{Viscerosensory}


Relating to corporeal information propagated by the spinal cord to brain regions involved in the sensation and coordination of bodily functions and associated behaviours.

\section{Iowa Gambling Task}

A test of reward processing that challenges the participant to maximize their earnings by forgoing short-term gains in return for eventual long-term gains.

\section{Total sleep deprivation}

The state associated with a complete absence of sleep in the prior night or nights.

\section{Balloon Analogue Risk Task}

(BART). A computerized measure of risk-taking behaviour. Participants are rewarded for inflating a 'balloon' but lose their reward if they overinflate and 'burst' the balloon.

\section{Wake propensity}

The likelihood of an organism maintaining the state of sustained wakefulness.

\section{Adenosine}

An endogenous compound involved in biochemical and neuromodulatory processes, including metabolism and sleep-wake regulation. It accumulates extracellularly with time spent awake and dissipates during slow-wave sleep.

\section{Sleep quality}

Measured subjectively, by means of self-reported perception of prior sleep, and objectively, using sleep-stage or quantitative electroencephalography sleep measures.

\section{Rapid eye movement}

(REM). Also known as paradoxical sleep. Sleep characterized by high-frequency, lowamplitude desynchronized electroencephalogram (particularly in the theta band), rapid eye movements, muscle paralysis and dreaming.

\section{Non-rapid eye movement}

(NREM). A type of sleep that consists of sleep stages 1-4 and that occurs towards the beginning of a sleep episode, and reflects homeostatic sleep processes.

\section{Slow-wave sleep}

Stage 3 and stage 4 of non-rapid eye movement sleep that is characterized by low-frequency ( $\sim 0.8-4 \mathrm{~Hz})$, high-amplitude synchronized electroencephalogram (called delta waves).

\section{Sleep spindles}

Synchronized phasic bursts of electrical activity often measured in the cortex or scalp surface electroencephalogram lasting several seconds in the 11-15 Hz frequency range.

\section{References}

1. Benca RM. Sleep in psychiatric disorders. Neurol Clin. 1996; 14:739-764. [PubMed: 8923493]

2. Wulff K, Gatti S, Wettstein JG, Foster RG. Sleep and circadian rhythm disruption in psychiatric and neurodegenerative disease. Nat Rev Neurosci. 2010; 11:589-599. [PubMed: 20631712] 
3. Centers for Disease Control and Prevention. Insufficient sleep is a public health problem. CDC; 2015. https://www.cdc.gov/features/dssleep

4. Durmer JS, Dinges DF. Neurocognitive consequences of sleep deprivation. Semin Neurol. 2005; 25:117-129. [PubMed: 15798944]

5. Van Dongen HP, Maislin G, Mullington JM, Dinges DF. The cumulative cost of additional wakefulness: dose-response effects on neurobehavioral functions and sleep physiology from chronic sleep restriction and total sleep deprivation. Sleep. 2003; 26:117-126. This article provides a comparison of the cumulative, dose-dependent deficits in sustained attention resulting from chronic sleep restriction of varied amount, relative to total SD. [PubMed: 12683469]

6. Belenky G, et al. Patterns of performance degradation and restoration during sleep restriction and subsequent recovery: a sleep dose-response study. J Sleep Res. 2003; 12:1-12. [PubMed: 12603781]

7. Borbély AA. A two process model of sleep regulation. Hum Neurobiol. 1982; 1:195-204. [PubMed: 7185792]

8. Goel N, Basner M, Rao H, Dinges DF. Circadian rhythms, sleep deprivation, and human performance. Prog Mol Biol Transl Sci. 2013; 119:155. [PubMed: 23899598]

9. Chee MW, et al. Effects of sleep deprivation on cortical activation during directed attention in the absence and presence of visual stimuli. Neuroimage. 2011; 58:595-604. [PubMed: 21745579]

10. Chee MW, Tan JC. Lapsing when sleep deprived: neural activation characteristics of resistant and vulnerable individuals. Neuroimage. 2010; 51:835-843. This paper describes the differences in fMRI signal in frontoparietal attention networks, thalamus and extrastriate cortex that differentiate individuals who are vulnerable (or, conversely, resilient) to impairments in attention following acute SD. [PubMed: 20171288]

11. Chee MW, Tan JC, Parimal S, Zagorodnov V. Sleep deprivation and its effects on object-selective attention. Neuroimage. 2010; 49:1903-1910. [PubMed: 19761853]

12. Chee MW, et al. Lapsing during sleep deprivation is associated with distributed changes in brain activation. J Neurosci. 2008; 28:5519-5528. [PubMed: 18495886]

13. Czisch M, et al. On the need of objective vigilance monitoring: effects of sleep loss on target detection and task-negative activity using combined EEG/fMRI. Front Neurol. 2012; 3:67. [PubMed: 22557992]

14. Drummond SP, et al. Sleep deprivation-induced reduction in cortical functional response to serial subtraction. Neuroreport. 1999; 10:3745-3748. [PubMed: 10716202]

15. Thomas M, et al. Neural basis of alertness and cognitive performance impairments during sleepiness. I. Effects of $24 \mathrm{~h}$ of sleep deprivation on waking human regional brain activity. J Sleep Res. 2000; 9:335-352. [PubMed: 11123521]

16. Tomasi $\mathrm{D}$, et al. Impairment of attentional networks after 1 night of sleep deprivation. Cereb Cortex. 2009; 19:233-240. [PubMed: 18483003]

17. Kong D, Soon CS, Chee MW. Functional imaging correlates of impaired distractor suppression following sleep deprivation. Neuroimage. 2012; 61:50-55. [PubMed: 22426349]

18. Mander BA, et al. Sleep deprivation alters functioning within the neural network underlying the covert orienting of attention. Brain Res. 2008; 1217:148-156. [PubMed: 18511023]

19. Muto V, et al. Local modulation of human brain responses by circadian rhythmicity and sleep debt. Science. 2016; 353:687-690. [PubMed: 27516598]

20. Chee MW, Choo WC. Functional imaging of working memory after $24 \mathrm{hr}$ of total sleep deprivation. J Neurosci. 2004; 24:4560-4567. This article provides a characterization of the changes in fMRI signal activity associated with working-memory impairments following acute $\mathrm{SD}$, and how these patterns change as a function of task complexity. [PubMed: 15140927]

21. Choo WC, Lee WW, Venkatraman V, Sheu FS, Chee MW. Dissociation of cortical regions modulated by both working memory load and sleep deprivation and by sleep deprivation alone. Neuroimage. 2005; 25:579-587. [PubMed: 15784437]

22. Habeck C, et al. An event-related fMRI study of the neurobehavioral impact of sleep deprivation on performance of a delayed-match-to-sample task. Brain Res Cogn Brain Res. 2004; 18:306-321. [PubMed: 14741317]

23. Portas CM, et al. A specific role for the thalamus in mediating the interaction of attention and arousal in humans. J Neurosci. 1998; 18:8979-8989. [PubMed: 9787003] 
24. Doran SM, Van Dongen HP, Dinges DF. Sustained attention performance during sleep deprivation: evidence of state instability. Arch Ital Biol. 2001; 139:253-267. [PubMed: 11330205]

25. Drummond SP, et al. The neural basis of the psychomotor vigilance task. Sleep. 2005; 28:10591068. [PubMed: 16268374]

26. Sridharan D, Levitin DJ, Menon V. A critical role for the right fronto-insular cortex in switching between central-executive and default-mode networks. Proc Natl Acad Sci USA. 2008; 105:12569-12574. [PubMed: 18723676]

27. Ma N, Dinges DF, Basner M, Rao H. How acute total sleep loss affects the attending brain: a metaanalysis of neuroimaging studies. Sleep. 2015; 38:233-240. [PubMed: 25409102]

28. Gumenyuk V, et al. Habitual short sleep impacts frontal switch mechanism in attention to novelty. Sleep. 2011; 34:1659-1670. [PubMed: 22131603]

29. Gazes Y, et al. Dual-tasking alleviated sleep deprivation disruption in visuomotor tracking: an fMRI study. Brain Cogn. 2012; 78:248-256. [PubMed: 22305924]

30. Drummond SP, Anderson DE, Straus LD, Vogel EK, Perez VB. The effects of two types of sleep deprivation on visual working memory capacity and filtering efficiency. PLoS ONE. 2012; 7:e35653. [PubMed: 22530064]

31. Turner TH, Drummond SP, Salamat JS, Brown GG. Effects of $42 \mathrm{hr}$ of total sleep deprivation on component processes of verbal working memory. Neuropsychology. 2007; 21:787-795. [PubMed: 17983292]

32. Chee MW, Chuah YM. Functional neuroimaging and behavioral correlates of capacity decline in visual short-term memory after sleep deprivation. Proc Natl Acad Sci USA. 2007; 104:9487-9492. [PubMed: 17517619]

33. Lythe KE, Williams SC, Anderson C, Libri V, Mehta MA. Frontal and parietal activity after sleep deprivation is dependent on task difficulty and can be predicted by the fMRI response after normal sleep. Behav Brain Res. 2012; 233:62-70. [PubMed: 22565029]

34. Chengyang L, , et al. Short-term memory deficits correlate with hippocampal-thalamic functional connectivity alterations following acute sleep restriction. Brain Imaging Behav. 2016.

35. Lei Y, et al. Large-scale brain network coupling predicts total sleep deprivation effects on cognitive capacity. PLoS ONE. 2015; 10:e0133959. [PubMed: 26218521]

36. Yoo S, Hu P, Gujar N, Jolesz F, Walker M. A deficit in the ability to form new human memories without sleep. Nat Neurosci. 2007; 10:385-392. This paper characterizes the effects of acute SD on fMRI hippocampal encoding activity and function connectivity during fact-based learning. [PubMed: 17293859]

37. Luber B, et al. Remediation of sleep-deprivation-induced working memory impairment with fMRIguided transcranial magnetic stimulation. Cereb Cortex. 2008; 18:2077-2085. This report demonstrates that repetitive TMS applied to visual occipital cortex can partially rescue performance impairments in a visual working-memory task following acute SD. [PubMed: 18203694]

38. Luber B, et al. Extended remediation of sleep deprived-induced working memory deficits using fMRI-guided transcranial magnetic stimulation. Sleep. 2013; 36:857-871. [PubMed: 23729929]

39. Luber B, et al. Facilitation of performance in a working memory task with rTMS stimulation of the precuneus: frequency-and time-dependent effects. Brain Res. 2007; 1128:120-129. [PubMed: 17113573]

40. Volkow ND, et al. Evidence that sleep deprivation downregulates dopamine D2R in ventral striatum in the human brain. J Neurosci. 2012; 32:6711-6717. This PET ligand-binding study in humans provides evidence of dopamine D2/3R downregulation in the striatum following acute SD. [PubMed: 22573693]

41. Tufik S. Changes of response to dopaminergic drugs in rats submitted to REM-sleep deprivation. Psychopharmacology. 1981; 72:257-260. [PubMed: 6111823]

42. Mullin BC, et al. Sleep deprivation amplifies striatal activation to monetary reward. Psychol Med. 2013; 43:2215-2225. [PubMed: 23286303]

43. Venkatraman V, Chuah YM, Huettel SA, Chee MW. Sleep deprivation elevates expectation of gains and attenuates response to losses following risky decisions. Sleep. 2007; 30:603-609. [PubMed: $17552375]$ 
44. Libedinsky $\mathrm{C}$, et al. Sleep deprivation alters valuation signals in the ventromedial prefrontal cortex. Front Behav Neurosci. 2011; 5:70. [PubMed: 22028686]

45. Olson EA, Weber M, Rauch SL, Killgore WD. Daytime sleepiness is associated with reduced integration of temporally distant outcomes on the Iowa Gambling Task. Behav Sleep Med. 2016; 14:200-211. [PubMed: 25396689]

46. Killgore WD, Balkin TJ, Wesensten NJ. Impaired decision making following $49 \mathrm{~h}$ of sleep deprivation. J Sleep Res. 2006; 15:7-13. [PubMed: 16489997]

47. Gujar N, Yoo SS, Hu P, Walker MP. Sleep deprivation amplifies reactivity of brain reward networks, biasing the appraisal of positive emotional experiences. J Neurosci. 2011; 31:44664474. [PubMed: 21430147]

48. Greer SM, Goldstein AN, Walker MP. The impact of sleep deprivation on food desire in the human brain. Nat Commun. 2013; 4:2259. This paper reports decreased fMRI signal in insular and frontal cortex, and increased amygdala activity, in response to desirable, high-calorie food item choices following acute SD. [PubMed: 23922121]

49. Menz MM, Buchel C, Peters J. Sleep deprivation is associated with attenuated parametric valuation and control signals in the midbrain during value-based decision making. J Neurosci. 2012; 32:6937-6946. [PubMed: 22593062]

50. Franken IH, van Strien JW, Nijs I, Muris P. Impulsivity is associated with behavioral decisionmaking deficits. Psychiatry Res. 2008; 158:155-163. [PubMed: 18215765]

51. Bechara A, Tranel D, Damasio H. Characterization of the decision-making deficit of patients with ventromedial prefrontal cortex lesions. Brain. 2000; 123:2189-2202. [PubMed: 11050020]

52 . Cedernaes $\mathbf{J}$, et al. Increased impulsivity in response to food cues after sleep loss in healthy young men. Obesity. 2014; 22:1786-1791. [PubMed: 24839251]

53. Demos K, et al. Partial sleep deprivation impacts impulsive action but not impulsive decisionmaking. Physiol Behav. 2016; 164:214-219. [PubMed: 27267950]

54. Acheson A, Richards JB, de Wit H. Effects of sleep deprivation on impulsive behaviors in men and women. Physiol Behav. 2007; 91:579-587. [PubMed: 17477941]

55. Ayalon L, Ancoli-Israel S, Drummond S. Altered brain activation during response inhibition in obstructive sleep apnea. J Sleep Res. 2009; 18:204-208. [PubMed: 19302344]

56. Anderson C, Platten CR. Sleep deprivation lowers inhibition and enhances impulsivity to negative stimuli. Behav Brain Res. 2011; 217:463-466. [PubMed: 20888369]

57. Libedinsky $\mathrm{C}$, et al. Sleep deprivation alters effort discounting but not delay discounting of monetary rewards. Sleep. 2013; 36:899-904. [PubMed: 23729933]

58. Rossa KR, Smith SS, Allan AC, Sullivan KA. The effects of sleep restriction on executive inhibitory control and affect in young adults. J Adolesc Health. 2014; 55:287-292. [PubMed: 24602612]

59. Womack SD, Hook JN, Reyna SH, Ramos M. Sleep loss and risk-taking behavior: a review of the literature. Behav Sleep Med. 2013; 11:343-359. [PubMed: 23286526]

60. Greer SM, Goldstein AN, Knutson B, Walker MP. A genetic polymorphism of the human dopamine transporter determines the impact of sleep deprivation on brain responses to rewards and punishments. J Cogn Neurosci. 2016; 28:803-810. [PubMed: 26918589]

61. Perogamvros L, Schwartz S. The roles of the reward system in sleep and dreaming. Neurosci Biobehav Rev. 2012; 36:1934-1951. [PubMed: 22669078]

62. McCann UD, et al. Effects of catecholamine depletion on alertness and mood in rested and sleep deprived normal volunteers. Neuropsychopharmacology. 1993; 8:345-356. [PubMed: 8099791]

63. Curb JD, Schneider K, Taylor JO, Maxwell M, Shulman N. Antihypertensive drug side effects in the Hypertension Detection and Follow-up Program. Hypertension. 1988; 11:II51-II55. [PubMed: 3350594]

64. Hershey T, Chad J. Effect of sleep deprivation on brain metabolism of depressed patients. Am J Psychiatry. 1992; 1:539.

65. Tomasi D, Wang GJ, Volkow N. Involvement of striatal dopamine D2/D3 receptors in the modulation of visual attention during rested wakefulness and sleep deprivation. Neuropsychopharmacology. 2015; 40:S247-S248. 
66. Wu JC, et al. Frontal lobe metabolic decreases with sleep deprivation not totally reversed by recovery sleep. Neuropsychopharmacology. 2006; 31:2783-2792. [PubMed: 16880772]

67. Elmenhorst $\mathrm{D}$, et al. Sleep deprivation increases $\mathrm{A}_{1}$ adenosine receptor binding in the human brain: a positron emission tomography study. J Neurosci. 2007; 27:2410-2415. This human PET ligandbinding study demonstrates adenosine $\mathrm{A}_{1}$ receptor upregulation in numerous cortical regions (maximal in OFC) and subcortical striatum following acute SD. [PubMed: 17329439]

68. Bonaventura $\mathrm{J}$, et al. Allosteric interactions between agonists and antagonists within the adenosine $\mathrm{A}_{2 \mathrm{~A}}$ receptor-dopamine $\mathrm{D}_{2}$ receptor heterotetramer. Proc Natl Acad Sci USA. 2015; 112:E3609E3618. [PubMed: 26100888]

69. Richard JM, Berridge KC. Nucleus accumbens dopamine/glutamate interaction switches modes to generate desire versus dread: $\mathrm{D}_{1}$ alone for appetitive eating but $\mathrm{D}_{1}$ and $\mathrm{D}_{2}$ together for fear. $\mathrm{J}$ Neurosci. 2011; 31:12866-12879. [PubMed: 21900565]

70. Park K, Volkow ND, Pan Y, Du C. Chronic cocaine dampens dopamine signaling during cocaine intoxication and unbalances $\mathrm{D}_{1}$ over $\mathrm{D}_{2}$ receptor signaling. J Neurosci. 2013; 33:15827-15836. [PubMed: 24089490]

71. Berro L, et al. Sleep deprivation impairs the extinction of cocaine-induced environmental conditioning in mice. Pharmacol Biochem Behav. 2014; 124:13-18. [PubMed: 24836180]

72. Brondel L, Romer MA, Nougues PM, Touyarou P, Davenne D. Acute partial sleep deprivation increases food intake in healthy men. Am J Clin Nutr. 2010; 91:1550-1559. [PubMed: 20357041]

73. Knutson B, Gibbs SE. Linking nucleus accumbens dopamine and blood oxygenation. Psychopharmacology. 2007; 191:813-822. [PubMed: 17279377]

74. Horne JA. Sleep function, with particular reference to sleep deprivation. Ann Clin Res. 1985; 17:199-208. [PubMed: 3909914]

75. Dinges DF, et al. Cumulative sleepiness, mood disturbance, and psychomotor vigilance performance decrements during a week of sleep restricted to 4-5 hours per night. Sleep. 1997; 20:267-277. This article provides a detailed behavioural characterization of the cognition and affective impact of cumulative, extended sleep restriction using subjective and objective measures. [PubMed: 9231952]

76. Zohar D, Tzischinsky O, Epstein R, Lavie P. The effects of sleep loss on medical residents' emotional reactions to work events: a cognitive-energy model. Sleep. 2005; 28:47-54. [PubMed: 15700720]

77. Minkel JD, et al. Sleep deprivation and stressors: evidence for elevated negative affect in response to mild stressors when sleep deprived. Emotion. 2012; 12:1015-1020. [PubMed: 22309720]

78. Bernert RA, Joiner TE. Sleep disturbances and suicide risk: a review of the literature. Neuropsychiatr Dis Treat. 2007; 3:735-743. [PubMed: 19300608]

79. Kamphuis J, Meerlo P, Koolhaas JM, Lancel M. Poor sleep as a potential causal factor in aggression and violence. Sleep Med. 2012; 13:327-334. [PubMed: 22305407]

80. Stubbs B, Wu YT, Prina AM, Leng Y, Cosco TD. A population study of the association between sleep disturbance and suicidal behaviour in people with mental illness. J Psychiatr Res. 2016; 82:149-154. [PubMed: 27501141]

81. Yoo SS, Gujar N, Hu P, Jolesz FA, Walker MP. The human emotional brain without sleep - a prefrontal amygdala disconnect. Curr Biol. 2007; 17:R877-R878. This paper describes amplified amygdala fMRI activity in response to negative experiences following acute SD, further associated with a loss of top-down connectivity with the mPFC. [PubMed: 17956744]

82. Motomura $\mathrm{Y}$, et al. Sleep debt elicits negative emotional reaction through diminished amygdalaanterior cingulate functional connectivity. PLoS ONE. 2013; 8:e56578. [PubMed: 23418586]

83. Prather AA, Bogdan R, Hariri AR. Impact of sleep quality on amygdala reactivity, negative affect, and perceived stress. Psychosom Med. 2013; 75:350-358. [PubMed: 23592753]

84. Goldstein AN, Walker MP. The role of sleep in emotional brain function. Annu Rev Clin Psychol. 2014; 10:679. [PubMed: 24499013]

85. Chuah LY, et al. Sleep deprivation and interference by emotional distracters. Sleep. 2010; 33:13051313. [PubMed: 21061852]

86. Killgore WD. Self-reported sleep correlates with prefrontal-amygdala functional connectivity and emotional functioning. Sleep. 2013; 36:1597-1608. [PubMed: 24179291] 
87. Goldstein AN, et al. Tired and apprehensive: anxiety amplifies the impact of sleep loss on aversive brain anticipation. J Neurosci. 2013; 33:10607-10615. [PubMed: 23804084]

88. Franzen PL, Buysse DJ, Dahl RE, Thompson W, Siegle GJ. Sleep deprivation alters pupillary reactivity to emotional stimuli in healthy young adults. Biol Psychol. 2009; 80:300-305. [PubMed: 19041689]

89. Etkin A, Wager TD. Functional neuroimaging of anxiety: a meta-analysis of emotional processing in PTSD, social anxiety disorder, and specific phobia. Am J Psychiatry. 2007; 164:1476-1488. [PubMed: 17898336]

90. Simmons A, Strigo I, Matthews SC, Paulus MP, Stein MB. Anticipation of aversive visual stimuli is associated with increased insula activation in anxiety-prone subjects. Biol Psychiatry. 2006; 60:402-409. [PubMed: 16919527]

91. Simmons AN, et al. Anxiety positive subjects show altered processing in the anterior insula during anticipation of negative stimuli. Hum Brain Mapp. 2011; 32:1836-1846. [PubMed: 21181800]

92. Harvey AG, Murray G, Chandler RA, Soehner A. Sleep disturbance as transdiagnostic: consideration of neurobiological mechanisms. Clin Psychol Rev. 2011; 31:225-235. [PubMed: 20471738]

93. Van Der Helm E, Gujar N, Walker MP. Sleep deprivation impairs the accurate recognition of human emotions. Sleep. 2010; 33:335-342. [PubMed: 20337191]

94. Daniela T, et al. Lack of sleep affects the evaluation of emotional stimuli. Brain Res Bull. 2010; 82:104-108. [PubMed: 20117179]

95. Goldstein-Piekarski AN, Greer SM, Saletin JM, Walker MP. Sleep deprivation impairs the human central and peripheral nervous system discrimination of social threat. J Neurosci. 2015; 35:1013510145. This study characterizes a failure in the accurate discrimination of anti-social versus prosocial facial signals at the behaviourally, brain (salience-detection network) and peripheral autonomic nervous system levels following acute SD. [PubMed: 26180190]

96. Simon EB, et al. Losing neutrality: the neural basis of impaired emotional control without sleep. $\mathrm{J}$ Neurosci. 2015; 35:13194-13205. [PubMed: 26400948]

97. Alfarra R, Fins AI, Chayo I, Tartar JL. Changes in attention to an emotional task after sleep deprivation: neurophysiological and behavioral findings. Biol Psychol. 2015; 104:1-7. [PubMed: 25451382]

98. Guadagni V, Burles F, Ferrara M, Iaria G. The effects of sleep deprivation on emotional empathy. J Sleep Res. 2014; 23:657-663. [PubMed: 25117004]

99. Guadagni V, et al. The relationship between quality of sleep and emotional empathy. $\mathrm{J}$ Psychophysiol. 2016.

100. Craig AD. How do you feel? Interoception: the sense of the physiological condition of the body. Nat Rev Neurosci. 2002; 3:655-666. [PubMed: 12154366]

101. Minkel J, Htaik O, Banks S, Dinges D. Emotional expressiveness in sleep-deprived healthy adults. Behav Sleep Med. 2011; 9:5-14. [PubMed: 21218289]

102. Berger RH, Miller AL, Seifer R, Cares SR, LeBourgeois MK. Acute sleep restriction effects on emotion responses in 30- to 36-month-old children. J Sleep Res. 2012; 21:235-246. [PubMed: 21988087]

103. Schwarz JF, et al. Shortened night sleep impairs facial responsiveness to emotional stimuli. Biol Psychol. 2013; 93:41-44. [PubMed: 23357729]

104. McGlinchey EL, et al. The effect of sleep deprivation on vocal expression of emotion in adolescents and adults. Sleep. 2011; 34:1233. [PubMed: 21886361]

105. Porges SW. The polyvagal theory: phylogenetic substrates of a social nervous system. Int J Psychophysiol. 2001; 42:123-146. [PubMed: 11587772]

106. Hoedlmoser K, Kloesch G, Wiater A, Schabus M. Self-reported sleep patterns, sleep problems, and behavioral problems among school children aged 8-11 years. Somnologie (Berl ). 2010; 14:23-31. [PubMed: 23162377]

107. Baum KT, et al. Sleep restriction worsens mood and emotion regulation in adolescents. J Child Psychol Psychiatry. 2014; 55:180-190. [PubMed: 24889207]

108. Killgore WD, et al. Sleep deprivation reduces perceived emotional intelligence and constructive thinking skills. Sleep Med. 2008; 9:517-526. [PubMed: 17765011] 
109. Sadeh A, et al. Sleep and psychological characteristics of children on a psychiatric inpatient unit. J Am Acad Child Adolesc Psychiatry. 1995; 34:813-819. [PubMed: 7608056]

110. Gordon AM, Chen S. The role of sleep in interpersonal conflict: do sleepless nights mean worse fights? Soc Psychol Personal Sci. 2014; 5:168-175.

111. Mallick BN, Singh A. REM sleep loss increases brain excitability: role of noradrenaline and its mechanism of action. Sleep Med Rev. 2011; 15:165-178. [PubMed: 21482157]

112. Siegel JM, Rogawski MA. A function for REM sleep: regulation of noradrenergic receptor sensitivity. Brain Res. 1988; 472:213-233. [PubMed: 3066435]

113. Kametani H, Kawamura H. Alterations in acetylcholine release in the rat hippocampus during sleep-wakefulness detected by intracerebral dialysis. Life Sci. 1990; 47:421-426. [PubMed: 2395411]

114. Marrosu F, et al. Microdialysis measurement of cortical and hippocampal acetylcholine release during sleep-wake cycle in freely moving cats. Brain Res. 1995; 671:329-332. [PubMed: 7743225]

115. Abel T, Havekes R, Saletin JM, Walker MP. Sleep, plasticity and memory from molecules to whole-brain networks. Curr Biol. 2013; 23:R774-R788. [PubMed: 24028961]

116. McDermott CM, et al. Sleep deprivation causes behavioral, synaptic, and membrane excitability alterations in hippocampal neurons. J Neurosci. 2003; 23:9687-9695. [PubMed: 14573548]

117. Fernandes C, et al. Detrimental role of prolonged sleep deprivation on adult neurogenesis. Front Cell Neurosci. 2015; 9:140. [PubMed: 25926773]

118. Drummond SP, et al. Altered brain response to verbal learning following sleep deprivation. Nature. 2000; 403:655-657. This article provides evidence that bidirectional changes in fMRI cortical and subcortical activity predict performance impairments and performance compensation during verbal learning following acute SD. [PubMed: 10688201]

119. Van Der Werf YD, et al. Sleep benefits subsequent hippocampal functioning. Nat Neurosci. 2009; 12:122-123. [PubMed: 19151712]

120. Antonenko D, Diekelmann S, Olsen C, Born J, Molle M. Napping to renew learning capacity: enhanced encoding after stimulation of sleep slow oscillations. Eur J Neurosci. 2013; 37:11421151. [PubMed: 23301831]

121. Saletin JM, et al. Human hippocampal structure: a novel biomarker predicting mnemonic vulnerability to, and recovery from, sleep deprivation. J Neurosci. 2016; 36:2355-2363. [PubMed: 26911684]

122. Chuah LY, et al. Donepezil improves episodic memory in young individuals vulnerable to the effects of sleep deprivation. Sleep. 2009; 32:999-1010. [PubMed: 19725251]

123. Poh JH, Chee MW. Degradation of cortical representations during encoding following sleep deprivation. Neuroimage. 2017; 153:131-138. [PubMed: 28161311]

124. Gujar N, Yoo SS, Hu P, Walker MP. The unrested resting brain: sleep deprivation alters activity within the default-mode network. J Cogn Neurosci. 2010; 22:1637-1648. [PubMed: 19702469]

125. Pepeu G, Giovannini MG, Bracco L. Effect of cholinesterase inhibitors on attention. Chem Biol Interact. 2013; 203:361-364. [PubMed: 23047023]

126. Sato H, Hata Y, Masui H, Tsumoto T. A functional role of cholinergic innervation to neurons in the cat visual cortex. J Neurophysiol. 1987; 58:765-780. [PubMed: 3681394]

127. Chuah LY, Chee MW. Cholinergic augmentation modulates visual task performance in sleepdeprived young adults. J Neurosci. 2008; 28:11369-11377. [PubMed: 18971479]

128. Mander BA, Winer JR, Jagust WJ, Walker MP. Sleep: a novel mechanistic pathway, biomarker, and treatment target in the pathology of Alzheimer's disease? Trends Neurosci. 2016; 39:552566. [PubMed: 27325209]

129. Mander B, et al. Age-related impairments of memory and fast sleep spindles are mediated by deterioration of cortico-thalamic white matter pathways [abstract]. Sleep. 2012; 35:A23.

130. Frey DJ, Badia P, Wright KP. Inter- and intra-individual variability in performance near the circadian nadir during sleep deprivation. J Sleep Res. 2004; 13:305-315. This study characterizes the variability in behavioural performance across several task domains in response to SD, both within and between individuals. [PubMed: 15560765] 
131. Porkka-Heiskanen T, et al. Adenosine: a mediator of the sleep-inducing effects of prolonged wakefulness. Science. 1997; 276:1265-1268. [PubMed: 9157887]

132. Van Dongen HP, Belenky G, Krueger JM. A local, bottom-up perspective on sleep deprivation and neurobehavioral performance. Curr Top Med Chem. 2011; 11:2414-2422. [PubMed: 21906023]

133. Saper CB, Fuller PM, Pedersen NP, Lu J, Scammell TE. Sleep state switching. Neuron. 2010; 68:1023-1042. [PubMed: 21172606]

134. Van Dongen H, Baynard MD, Maislin G, Dinges DF. Systematic interindividual differences in neurobehavioral impairment from sleep loss: evidence of trait-like differential vulnerability. Sleep. 2004; 27:423-433. This behavioural study describes inter-individual differences in behavioural task impairments following SD and how these relate to prior sleep history of those individuals. [PubMed: 15164894]

135. Mollicone DJ, Van Dongen H, Rogers NL, Banks S, Dinges DF. Time of day effects on neurobehavioral performance during chronic sleep restriction. Aviat Space Environ Med. 2010; 81:735-744. [PubMed: 20681233]

136. Rupp TL, Wesensten NJ, Balkin TJ. Trait-like vulnerability to total and partial sleep loss. Sleep. 2012; 35:1163-1172. [PubMed: 22851812]

137. Kuna ST, et al. Heritability of performance deficit accumulation during acute sleep deprivation in twins. Sleep. 2012; 35:1223-1233. [PubMed: 22942500]

138. Basner M, Rao H, Goel N, Dinges DF. Sleep deprivation and neurobehavioral dynamics. Curr Opin Neurobiol. 2013; 23:854-863. [PubMed: 23523374]

139. Cui J, et al. Microstructure of frontoparietal connections predicts individual resistance to sleep deprivation. Neuroimage. 2015; 106:123-133. [PubMed: 25463450]

140. Vandewalle G, et al. Functional magnetic resonance imaging-assessed brain responses during an executive task depend on interaction of sleep homeostasis, circadian phase, and PER3 genotype. J Neurosci. 2009; 29:7948-7956. [PubMed: 19553435]

141. Marcus ER. Two views of brain function. Trends Cogn Sci. 2010; 14:180-190. [PubMed: 20206576]

142. Fox MD, Raichle ME. Spontaneous fluctuations in brain activity observed with functional magnetic resonance imaging. Nat Rev Neurosci. 2007; 8:700-711. [PubMed: 17704812]

143. Yeo BT, Tandi J, Chee MW. Functional connectivity during rested wakefulness predicts vulnerability to sleep deprivation. Neuroimage. 2015; 111:147-158. [PubMed: 25700949]

144. Kaufmann T, et al. The brain functional connectome is robustly altered by lack of sleep. Neuroimage. 2016; 127:324-332. [PubMed: 26712339]

145. Gao L, et al. Frequency-dependent changes of local resting oscillations in sleep-deprived brain. PLoS ONE. 2015; 10:e0120323. [PubMed: 25798918]

146. Wang Y, Liu H, Hitchman G, Lei X. Module number of default mode network: inter-subject variability and effects of sleep deprivation. Brain Res. 2015; 1596:69-78. [PubMed: 25446443]

147. Fox MD, et al. The human brain is intrinsically organized into dynamic, anticorrelated functional networks. Proc Natl Acad Sci USA. 2005; 102:9673-9678. [PubMed: 15976020]

148. De Havas JA, Parimal S, Soon CS, Chee MW. Sleep deprivation reduces default mode network connectivity and anti-correlation during rest and task performance. Neuroimage. 2012; 59:17451751. [PubMed: 21872664]

149. Bosch OG, et al. Sleep deprivation increases dorsal nexus connectivity to the dorsolateral prefrontal cortex in humans. Proc Natl Acad Sci USA. 2013; 110:19597-19602. [PubMed: 24218598]

150. Sämann PG, et al. Increased sleep pressure reduces resting state functional connectivity. MAGMA. 2010; 23:375-389. [PubMed: 20473549]

151. Shao Y, et al. Decreased thalamocortical functional connectivity after 36 hours of total sleep deprivation: evidence from resting state fMRI. PLoS ONE. 2013; 8:e78830. [PubMed: 24205327]

152. Shao $\mathrm{Y}$, et al. Altered resting-state amygdala functional connectivity after 36 hours of total sleep deprivation. PLoS ONE. 2014; 9:e112222. [PubMed: 25372882] 
153. Leech R, Sharp DJ. The role of the posterior cingulate cortex in cognition and disease. Brain. 2014; 137:12-32. [PubMed: 23869106]

154. Tagliazucchi E, et al. Large-scale brain functional modularity is reflected in slow electroencephalographic rhythms across the human non-rapid eye movement sleep cycle. Neuroimage. 2013; 70:327-339. [PubMed: 23313420]

155. Vyazovskiy VV, et al. Local sleep in awake rats. Nature. 2011; 472:443-447. [PubMed: 21525926]

156. Horovitz SG, et al. Decoupling of the brain's default mode network during deep sleep. Proc Natl Acad Sci USA. 2009; 106:11376-11381. [PubMed: 19549821]

157. Brower KJ, Aldrich MS, Robinson EA, Zucker RA, Greden JF. Insomnia, self-medication, and relapse to alcoholism. Am J Psychiatry. 2001; 158:399-404. [PubMed: 11229980]

158. Thomas RJ, Rosen BR, Stern CE, Weiss JW, Kwong KK. Functional imaging of working memory in obstructive sleep-disordered breathing. J Appl Physiol (1985). 2005; 98:2226-2234. [PubMed: 15677733]

159. Wong MM, Brower KJ, Fitzgerald HE, Zucker RA. Sleep problems in early childhood and early onset of alcohol and other drug use in adolescence. Alcohol Clin Exp Res. 2004; 28:578-587. [PubMed: 15100609]

160. Van Cauter E, et al. Impact of sleep and sleep loss on neuroendocrine and metabolic function. Horm Res. 2007; 67(Suppl 1):2-9. [PubMed: 17308390]

161. Markwald RR, et al. Impact of insufficient sleep on total daily energy expenditure, food intake, and weight gain. Proc Natl Acad Sci USA. 2013; 110:5695-5700. [PubMed: 23479616]

162. Michaelides M, et al. PET imaging predicts future body weight and cocaine preference. Neuroimage. 2012; 59:1508-1513. [PubMed: 21889993]

163. Germain A. Sleep disturbances as the hallmark of PTSD: where are we now? Am J Psychiatry. 2013; 170:372-382. [PubMed: 23223954]

164. Mellman TA, Nolan B, Hebding J, Kulick-Bell R, Dominguez R. A polysomnographic comparison of veterans with combat-related PTSD, depressed men, and non-ill controls. Sleep. 1997; 20:46-51. [PubMed: 9130334]

165. Lavie P, Hefez A, Halperin G, Enoch D. Long-term effects of traumatic war-related events on sleep. Am J Psychiatry. 1979; 136:175-178. [PubMed: 216274]

166. Breslau N, et al. Sleep in lifetime posttraumatic stress disorder: a community-based polysomnographic study. Arch Gen Psychiatry. 2004; 61:508-516. [PubMed: 15123496]

167. Habukawa M, Uchimura N, Maeda M, Kotorii N, Maeda H. Sleep findings in young adult patients with posttraumatic stress disorder. Biol Psychiatry. 2007; 62:1179-1182. [PubMed: 17543289]

168. Mellman TA, Bustamante V, Fins AI, Pigeon WR, Nolan B. REM sleep and the early development of posttraumatic stress disorder. Am J Psychiatry. 2002; 159:1696-1701. [PubMed: 12359675]

169. Mellman TA, Kumar A, Kulick-Bell R, Kumar M, Nolan B. Nocturnal/daytime urine noradrenergic measures and sleep in combat-related PTSD. Biol Psychiatry. 1995; 38:174-179. [PubMed: 7578660]

170. Calohan J, Peterson K, Peskind ER, Raskind MA. Prazosin treatment of trauma nightmares and sleep disturbance in soldiers deployed in Iraq. J Trauma Stress. 2010; 23:645-648. [PubMed: 20931662]

171. Raskind MA, et al. Reduction of nightmares and other PTSD symptoms in combat veterans by prazosin: a placebo-controlled study. Am J Psychiatry. 2003; 160:371-373. [PubMed: 12562588]

172. Raskind MA, et al. A parallel group placebo controlled study of prazosin for trauma nightmares and sleep disturbance in combat veterans with posttraumatic stress disorder. Biol Psychiatry. 2007; 61:928-934. [PubMed: 17069768]

173. Taylor FB, et al. Prazosin effects on objective sleep measures and clinical symptoms in civilian trauma posttraumatic stress disorder: a placebo-controlled study. Biol Psychiatry. 2008; 63:629632. [PubMed: 17868655]

174. Jovanovic T, et al. Posttraumatic stress disorder may be associated with impaired fear inhibition: relation to symptom severity. Psychiatry Res. 2009; 167:151-160. [PubMed: 19345420] 
175. Jovanovic T, Kazama A, Bachevalier J, Davis M. Impaired safety signal learning may be a biomarker of PTSD. Neuropharmacology. 2012; 62:695-704. [PubMed: 21377482]

176. Grillon C, Morgan CA III. Fear-potentiated startle conditioning to explicit and contextual cues in Gulf War veterans with posttraumatic stress disorder. J Abnorm Psychol. 1999; 108:134-142. [PubMed: 10066999]

177. Menz MM, et al. The role of sleep and sleep deprivation in consolidating fear memories. Neuroimage. 2013; 75:87-96. [PubMed: 23501052]

178. Spoormaker VI, et al. Effects of rapid eye movement sleep deprivation on fear extinction recall and prediction error signaling. Hum Brain Mapp. 2012; 33:2362-2376. [PubMed: 21826762]

179. Straus LD, Acheson DT, Risbrough VB, Drummond SP. Sleep deprivation disrupts recall of conditioned fear extinction. Biol Psychiatry Cogn Neurosci Neuroimaging. 2017; 2:123-129. [PubMed: 28758152]

180. Marshall AJ, Acheson DT, Risbrough VB, Straus LD, Drummond SP. Fear conditioning, safety learning, and sleep in humans. J Neurosci. 2014; 34:11754-11760. [PubMed: 25164670]

181. Greicius MD, Krasnow B, Reiss AL, Menon V. Functional connectivity in the resting brain: a network analysis of the default mode hypothesis. Proc Natl Acad Sci USA. 2003; 100:253-258. [PubMed: 12506194]

182. Spreng RN, Grady CL. Patterns of brain activity supporting autobiographical memory, prospection, and theory of mind, and their relationship to the default mode network. J Cogn Neurosci. 2010; 22:1112-1123. [PubMed: 19580387]

183. Spreng RN, Stevens WD, Chamberlain JP, Gilmore AW, Schacter DL. Default network activity, coupled with the frontoparietal control network, supports goal-directed cognition. Neuroimage. 2010; 53:303-317. [PubMed: 20600998] 

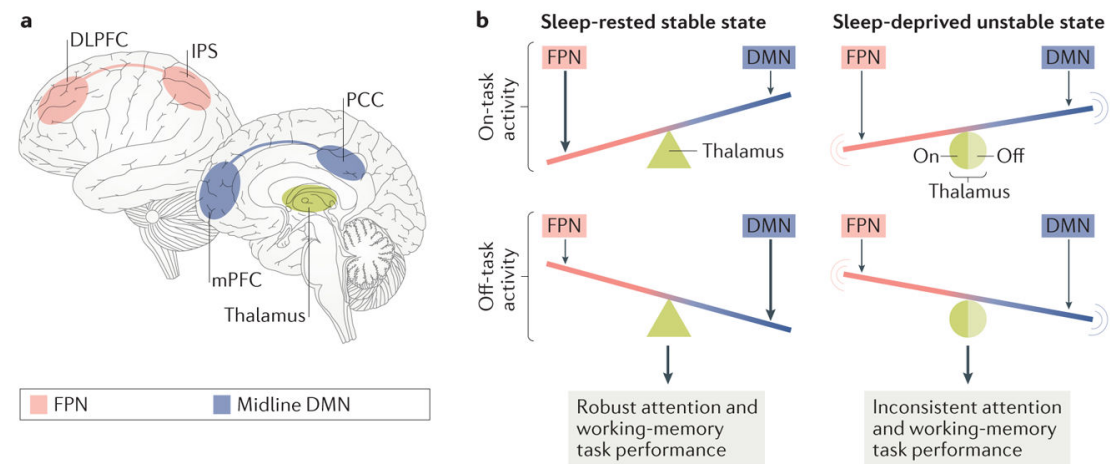

Figure 1. Sleep loss, attention and working memory

a Brain regions and networks associated with attention and working memory (frontoparietal network (FPN); red), arousal (thalamus; green) and the default mode network (DMN; blue) are affected by sleep deprivation. The DMN is a collection of brain areas, including midline frontoparietal regions, that often disengage when an individual performs an externally driven, goal-directed task and then re-engage when an individual stops performing that task. Suppression of the DMN is necessary to mobilize appropriate on-task brain networks to achieve successful goal-directed behaviour ${ }^{181}$, unless that task requires access to internally stored relevant information such as autobiographical memories or previously learned predictive cues ${ }^{18,182,183}$. Brain regions across multiple brain networks, including the dorsolateral prefrontal cortex (DLPFC), intraparietal sulcus (IPS), thalamus, medial prefrontal cortex (mPFC) and posterior cingulate cortex (PCC), are differentially altered by sleep loss. $\mathbf{b} \mid$ In the sleep-rested state, there is reciprocal inhibition between taskrelated FPN activity and DMN activity, supported by sustained ascending arousal input from the thalamus. This leads to consistent attentional and working-memory performance. In the sleep-deprived state, there is unstable reciprocal inhibition between task-related FPN activity and DMN activity, and erratic ascending arousal activity influencing thalamic activity. As a result, there is reduced task-related FPN activity and intermittent intrusions of DMN activity during task engagement. The behavioural consequence is variable and/or impaired attention and working-memory performance, worsening with lowered thalamic activity and improving with higher thalamic activity. 


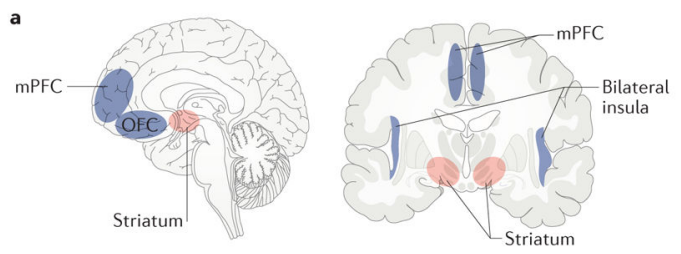

b

Sleep-rested (Low adenosine load)

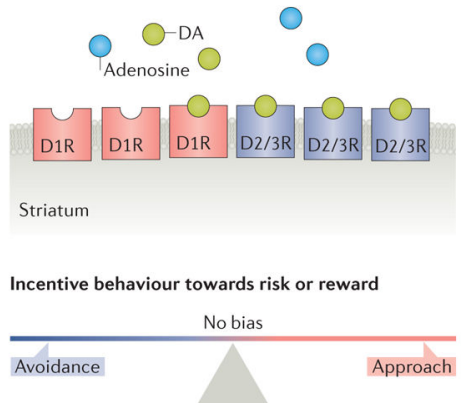

Sleep-rested

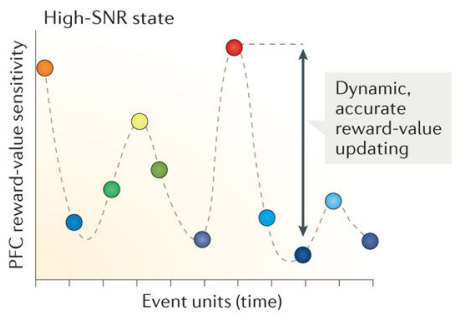

Sleep-deprived (High adenosine load)
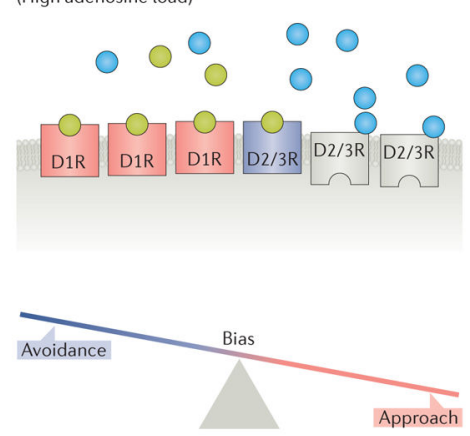

Sleep-deprived

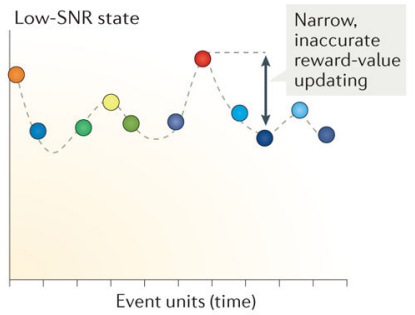

Reward probability

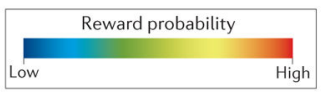

Figure 2. Sleep loss and incentive processing

a Reward-relevant brain regions that are affected by sleep deprivation (SD) include cortical regions (blue) such as the medial prefrontal cortex (mPFC), insula and orbitofrontal cortex (OFC), and the subcortical region of the striatum (red). $\mathbf{b} \mid$ Increased adenosine load (blue circles) associated with SD triggers downregulation of dopamine (DA) D2 and D3 receptors (D2/3Rs), resulting in decreased receptor membrane expression within the striatum (internalized receptors; grey). Consequently, there is a greater ratio of D1R to D2/3R availability, and the relative increase in striatal D1R activation by DA (green circles) under SD conditions is proposed to increase risk-related and reward-related approach behaviours, shown in the see-saw imbalance. $\mathbf{c} \mid$ SD further disrupts incentive processing within PFC regions and thus is proposed to result in a lower signal-to-noise ratio (SNR) under SD conditions (represented by a narrow dynamic range of PFC sensitivity). This consequentially impairs the ability to accurately map and update changing value or reward probability (coloured dots) over time - such as that involved in reinforcement learning, ultimately contributing to non-optimal incentive-based decision making. 

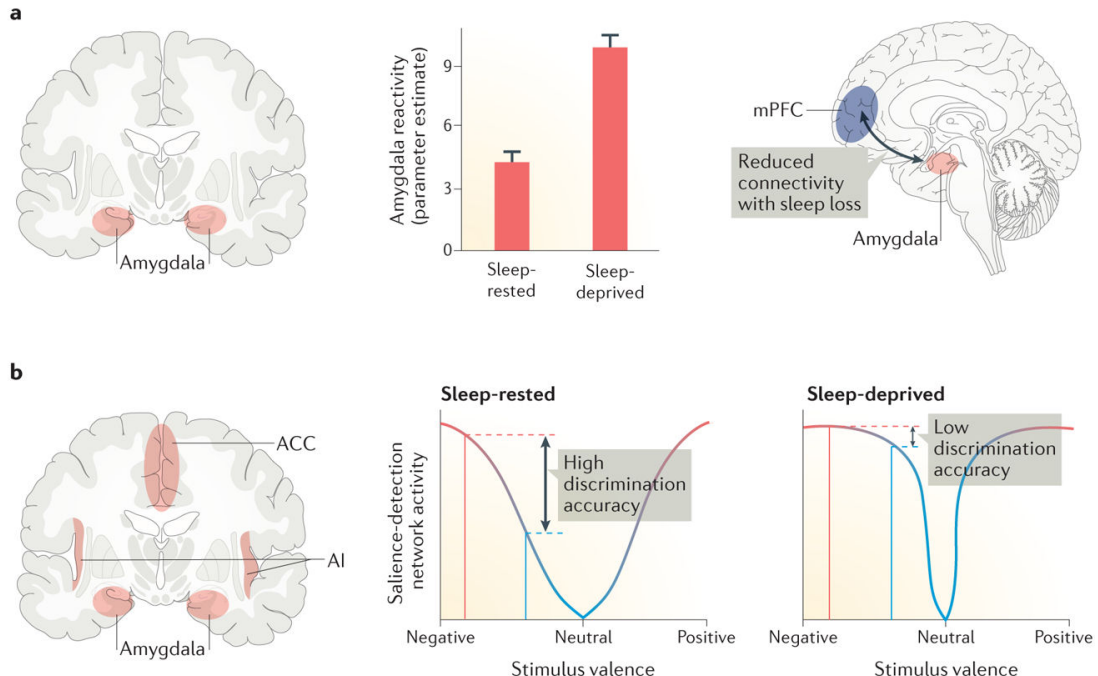

c

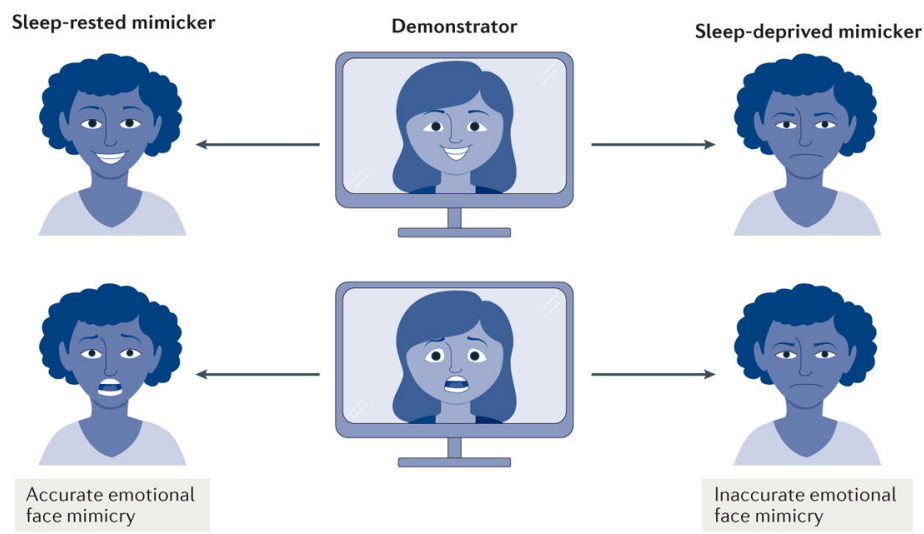

Figure 3. Sleep loss and aversive processing

a Sleep deprivation (SD) amplifies amygdala reactivity (red) in response to negative emotional stimuli and decreases associated amygdala-medial prefrontal cortex (mPFC) connectivity (blue). $\mathbf{b} \mid \mathrm{SD}$ alters sensitivity of the salience-detection network (the amygdala, anterior cingulate cortex (ACC) and anterior insula (AI)) to varying levels of emotional stimuli that range in valence strength ( $x$ axis in the two graphs) from negative (red) to neutral (blue) to positive (red). Under sleep-rested conditions (left graph), there is a wide and dynamic range of salience-detection sensitivity, resulting in the ability to accurately discriminate between degrees of emotional salience (tall vertical difference arrow; discerning emotional (red vertical line) from neutral stimuli (blue vertical line)). By contrast, $\mathrm{SD}$ (right graph) is proposed to trigger a narrowing and thus more nonspecific detection sensitivity range, impairing the ability to accurately discriminate between degrees of emotional salience (short vertical difference arrow). This loss of 'net neutrality' results in over-generalized emotional sensitivity, wherein an otherwise largely neutral stimulus (blue vertical line) is inappropriately registered as 'emotional' by the salience-detection network; further observed in biased emotional ratings of neutral stimuli. c $\mid$ A downstream behavioural consequence of these central brain changes, in combination with disrupted peripheral autonomic nervous system feedback of visceral body information, could lead to inaccurate 
and even absent outward expression of emotions. This is supported by experimental evidence demonstrating that individuals deprived of sleep fail to register emotional faces shown to them on a screen and consequently are unable to accurately mimic the emotional face expressions of these target faces themselves. Part $\mathbf{a}$ is adapted with permission from REF. 81, Elsevier. 


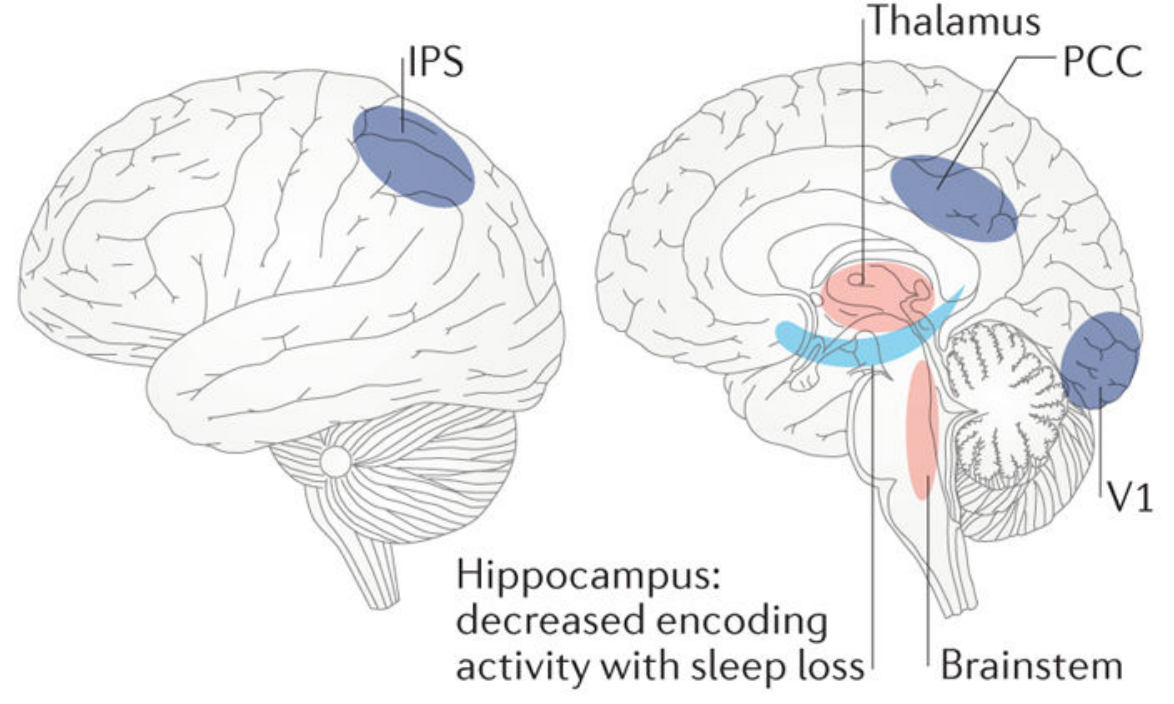

\section{Increased hippocampal connectivity with sleep loss Decreased hippocampal connectivity with sleep loss}

Figure 4. Sleep loss and hippocampal memory encoding

Sleep deprivation (SD) decreases encoding-related activity within the hippocampus (light blue), compared with normal sleep-rested conditions. Moreover, in sleep-deprived individuals, hippocampal connectivity with encoding-relevant cortical regions of the intraparietal sulcus (IPS), posterior cingulate cortex (PCC) and primary visual cortex (V1) (purple regions) during encoding is lower than in sleep-rested individuals. By contrast, hippocampal encoding-related connectivity with subcortical arousal regions of the thalamus and brainstem (red) after SD is increased. 